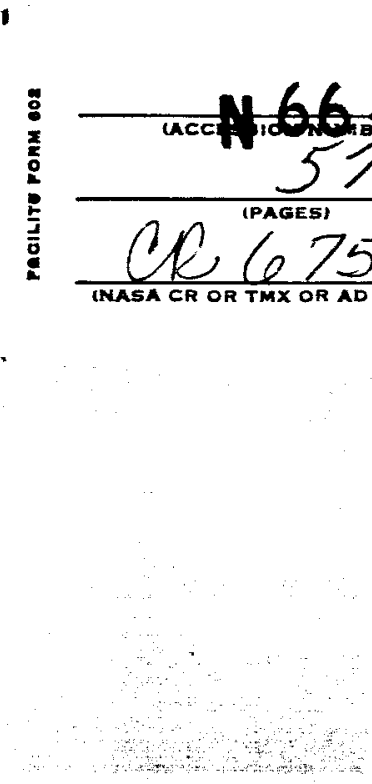

GPO PRICE

CFSTI PRICE(S)

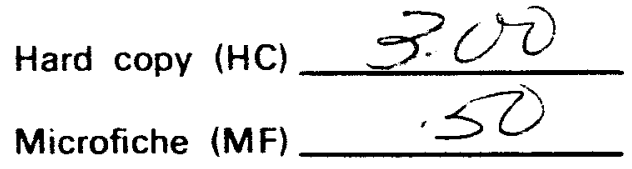

ff 653 July 65

\title{
SATELLITE MOTION FOR ALL INCLINATIONS AROUND AN OBLATE PLANET
}

AUGUST 1964

M. ECKSTEIN

DESEARCH SOECIALIST DOUGLAS A RCRAFT CO., INC.

Y. SHI

RESEARCH SPECIALIST DOUGLAS AIRCAAFT CO., INC.

J. KEVORKIAN CONSULTANT DOUGLAS AIRCRAFT CO.. INC.

TO gE PRESENTED TO THE IAL SYMPOSIUM AT THESSALONIKI, GREECE. ON AUGUST $17-22,1964$

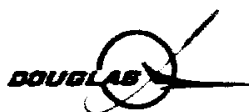

DOLGLAS MISSILE\& SPACE SYSTEMS DIVISION 
ERTATÁ FOA DOUGLLÁ PAPER NO. $307 \overline{8}$

SATELLITE MOTION FOR ALU INCLINATIONS AROUND AN OBLATE PLANET

Page Equation

Should Read

23

(3.5e)

$p=r^{2} \sin ^{2} \theta \frac{d}{d t}$

23

$(3.58)$

$\frac{d \varphi}{d t}=\frac{p u^{2}}{\cos i}+\frac{\cos ^{3} i \cos \theta}{p \sin ^{2} i \sin \theta}\left[\frac{\partial U}{\partial \theta}+\tan i \frac{\cos \phi}{\sin \theta} \frac{\partial U}{\partial \varphi}\right]$

26

(3.8a)

$1(\phi ; E)=\sum_{n=0} 1_{n / 2}\left(\phi_{i} \dot{\phi}_{i} \varepsilon\right) \cdot \varepsilon^{n / 2}$

26

(3. Bb)

$u(\phi ; \varepsilon)=\sum_{n=0} u_{n / 2}(\phi, \phi ; \varepsilon) \cdot \varepsilon^{n / 2}$

27

$(3.12 a)$

$\frac{\partial i_{1}}{\partial \phi}=\frac{d i_{00}}{\partial \dot{\partial}}-\frac{1}{p^{4}} \cos ^{5} 1_{00} \sin i_{\infty} \sin 2 \phi\left[1+e_{0} \cos (\varphi-\omega)\right]$

$1_{0}, \omega_{1}$, and $e_{1}$ :

(The asterisk refers to the note on next page)

30

Iine above

(3.20a)

at $t=\tau$ are

30

$(3.20 b)$

$e_{1}=n_{1}+\frac{B_{2}}{2 S_{0}}(\cos 2 w-\cos 2 w)$

33

$(3.29 \mathrm{c})$

$\frac{d w_{1 / 2}}{d \bar{C}}=\frac{1}{2} s_{2}\left(1_{01 / 2}\right)^{2}+s_{1} i_{01} \cdot+A_{0} \cdot+A_{2} \cdot \cos 2 \omega$

34

(3.33)

$1_{01 / 2}=\frac{1}{s_{1}}\left[ \pm\left(\bar{x}_{0}-x_{1} \cos 2 \omega\right)^{1 / 2}-s_{0}\right]$ 
Page Equat10n

34

34

34

(3.37)

36

(3.43)

37

38

38

38

39

(3.54a)

below

(3.49)

(3.50)

line abor

(3.49)

39 third line
below $(3.55)$

39

\section{Should Pead}

should have \pm on the right side next to the $=$ sign

should have \pm on the right side next to the $=$ sign

$$
\begin{aligned}
& e_{1}^{*}= \pm \frac{I}{x_{1}}\left(\bar{x}_{0}-x_{1} \cos 2 \omega^{\prime \prime}\right)\left[-\frac{\bar{s}_{0}}{s_{1}} B_{21}+E_{1 / 2} B_{22}\right.
\end{aligned}
$$

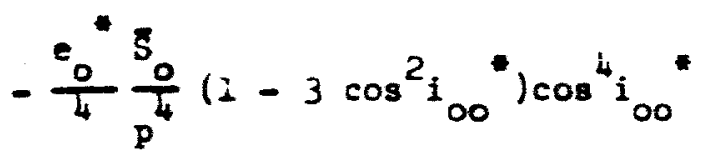

$$
\begin{aligned}
& -\frac{1}{2}\left[\frac{\mathrm{B}_{21}}{\mathrm{~S}_{1}}+\frac{\mathrm{B}_{21}{ }^{*} \mathrm{~B}_{2}^{*}}{\alpha_{1}}\right] \cos 2 \omega^{*}+\mathrm{E}_{1}
\end{aligned}
$$$$
i_{o c}=i_{o 0}^{\bullet}+\varepsilon^{1 / 2} i_{01 / 2}+\varepsilon_{01}
$$

- . expansions to $O(\varepsilon)$ for all inclinations... should have \pm on the right side next to the $=$ sign

$$
2 \sin \omega \cos \omega^{*}=\frac{d v}{d \omega}= \pm 2[v(1-v)]^{1 / 2}
$$

should have \pm in front of the integral

should have $t$ on the right side next to the = sign

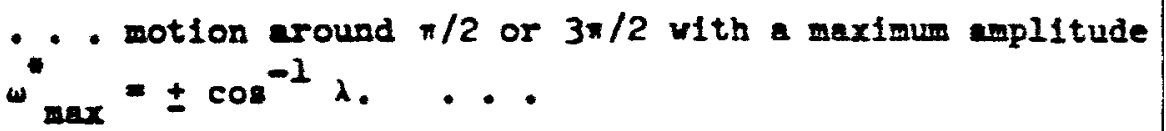$$
\lambda=\sin ^{2} w-\left(s_{0}+s_{1} j_{1 / 2}\right)^{2} / 2 x_{1}
$$ 
Page Equstion

40

40

(3.59)

$40 \quad(3.60)$

$40 \quad(3.61)$

40

(3.62)

40 lines below (3.62) down to the botton of the page

41

(3.64b)

$41 \quad(3.64 c)$

4. second line
of $(3.77)$

46

(3.84)

53

\section{Should Read}

$\overline{-}-\bar{t}= \pm \int \frac{d \omega}{\left(2 x_{1}\right)^{1 / 2} \sin \omega}= \pm \frac{1}{\left(2 x_{1}\right)^{1 / 2}} \log \left|\tan \frac{\omega}{2}\right|$

$\left|\tan \frac{\omega}{2}\right|=e^{ \pm\left(2 x_{1}\right)^{1 / 2}\left(-\bar{\sigma}_{0}\right)}$

$$
\lambda=\sin ^{2} w-\left(\bar{s}_{0}+s_{1} j_{1 / 2}\right)^{2} / 2 x_{1}=0
$$

$\sin w^{*}= \pm\left(\bar{S}_{0}-s_{1} J_{1 / 2}\right) /\left(2 \alpha_{1}\right)^{1 / 2}$

$v^{*}= \pm \sin ^{-1}\left[\left(\bar{S}_{0}-s_{1} j_{1 / 2}\right) /\left(2 k_{1}\right)^{1 / 2}\right]$

should be replaced by:

- - are assumed. This means that Case II happens only if initially the apse and the inclination are chosen properly as to satisfy the condition (3.62).

$k_{2}=\left[\frac{2 x_{1}}{x_{1}+\bar{x}_{0}}\right]^{1 / 2}$

should have + on the right side next to the = sign

$\left.-\cos 2 \varphi-\frac{e_{0}}{2} \cos \left(t+\omega_{c}\right)-\frac{e_{0}}{2} \cos \left(3--\omega_{c}\right)\right)$

$$
\Omega_{01}=D_{1} \omega_{0}+\int_{0}^{\omega_{c}} \ldots
$$

$g_{1}=-\frac{s_{2}}{2 s_{1}^{2}} \xi_{0}+\frac{1}{x_{1}}\left[-c_{21} s_{0}+c_{22} s_{1} E_{1 / 2}\right]$ 
SATELUTEE HOTYON ZOR ALL FHCUIHTIOHS

AROULID AII OBEATE PLAIIET*

M. Eckstein ${ }^{\dagger}, Y, \operatorname{Sni}^{+t}$ and J. Kevorkian ${ }^{++}$

ASTRACT

104

A uniformly valid soiution for the sotion of a sateilite around an obiate planet is presented. The Two Variable Expansion Trocedure as earier developed at Caitech uns applied to obtain a soiution valia for ali inclinations including the criticai. This solution is correct to oraer $\varepsilon$, where $\epsilon$ is a small parameter proportional to the oblateness parameter $J_{2}$. The reciprocai of the radius vector, eccentricity, perigee, inclination, and node of the satelite orbit are given as functions of the central angle between node and satelite. The results are based on a potential which inoiudes the second and rourth zonal harmonics. The solution for the case of eritical inclination is first ohtained separately and then ratched with the soiution of the noncritical case to establish solution uniformly valid for aii inclinetions.

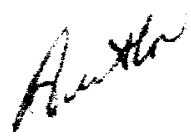

- This work was jointly sponsored by the liational Aeronautics and 5 pace Administration under Contract No. MASW-90I and the U. S. Air Force unaer Grant No. AF-AFOSR-62-256.

+ Research Specialist, Douglas Afrcraft Co., Inc., Santa Monica, Caifornia.

tt Reseerch Specialist, Dougles Aircraft Co., Ine., Santa Monica, California, Presently, Assistant Professor of Aeronautics, Carnegie Institute of Technoiogs. Pittsburgh, Pennsylvaria.

t+tConsultant, Douglas Aircraft Co., Inc.. Sante Monica, Californie nad Researcin fellow in Acronautics, Califoraia Institute of Technology, Pasadena, Caili. 


\section{Introduction}

The motion of a satellite around an oblate planet has receired considerable attention in the literature arter the advent of artificial satellites of the earth. The early theorles, of wich Brouver's (1959) 18 the post comprehensive, vere not ralid for intial orbital inclinations close to the critical value $\cos ^{-1}(5)^{-1 / 2}=63.4^{\circ}$ frow the equatorial plane of symetry. The non-validity of the solution at this angle exhibited itgelf by the occurrence of a divisor which tended to zero at the critical inclination.

Later, Hori (1960) and others (cf. Garfinkel (1960), Mergman (1962), and Izsak (1963) unlng diverse approsches, atudied the behavior of the solution near the critical Inclination. Though "a direct analytic conparison of the various treatweats of the critical Inclination problem is almost impossible because of the mutiplicity of notations, approximations and starting points" (Mersman (2962). there is general agreenent about the necessity of studying an expansion in powers of $J^{1 / 2}$ (where $J$ is the small parameter measuring the oblateness perturbations). Furthermore, at least the qualitative behavior of the motion near the critical inclination, as first described by Hori, has been repeatediy substantiated. This statement by Mersman quite correctly reflects the inherent algebraic complexity of the main problem and the necessarily inrolved nature of Its solution. However, the basic mathematical problem that gives rise to the singularity at the critical inclination is quite simple and was recognized by many authors. In partfcular, Struble (1961) has pointed out that for Inclinetions close to the critical the equations governing the slow variations of the apse and inclination angle are coupled by virtue of a regrouping of terms which otherwise have different or Jars of magnitude. 
This phenonenon can be cuplicated exactly in a particularly simple model equation corresponding to the forced oscillations of a systen with an eppropriate mall non-inearity. The conection betreen non-linear oscillations and satellite notions with swall pertumbations is, of course, vell known since it was first proposed by Laplace in his study of the notion of the moon. Therefore, in order to fix ideas the proposed model equation is first studied in detail, and the techniques are then directly spplied to the main problem. The aim of the present paper is to develop the solution both near and aray from the criticai inclination in asyaptotic series vith respect to $\mathrm{J}$. These series are uniformil valid for long times, but the primary gon is the achievement of uniform validity for all inclination angles as vell.

The approach adopted here proceeds from the formulation proposed by Struble (1960) and (2961). It is first bhown that two distinct asymptotic expansions (corresponding to two regines of the initial inclination near and away from the critical) can be constructed ard rendered uniformiy valid for long titej by the two-variabie ex arsion procesine of fievorian (i962). It is then demonstrated tinnt each of the above eeneraiized asymptotic expansions, depenaina upon the initial inclination, inaiviaually describe the notion for all times. In addition, the tro expansioas match in an overiap donain of the inclination paraneter lying between the critical and non-critical regines. This matching Is in the sense of the theory of Kapiun and Lagerstrom (1957), hence the uniformy valid asymptotic representation of the motion follows easily.

Furthernore, the analytic dependence of the solution on $\mathrm{J}^{1 / 2}$, as first sujsested by Hori (1960), is justified by the techniques of singuiar perturbation theory and the matching process. 
The present solution includes the second and fourth goral harmonics of the earth's potentiai. Al secular and long-period terms are included up to $O\left(J^{5 / 2}\right)$, while short-period terms are retained up to $O(J)$. The results are exhibited in the form of the reciprocal redius, eccentricity, perigee, inciination, and node as functions of the central angle between the ascendins node and radius vector. The equation for the time is not given here but will be inciuded in a future pubilication. A detailed ccuparison of the present results with at lesst the work of Struble (1961) and (1962) will aiso be provided there. 


\section{Niodei Equation}

\subsection{General Discussion}

In order to demonstrate the essential mathematical features of the main proiden and the expansion procedures, the foilowing model equation is first siudied in detaii

$$
\frac{a^{2} y}{d t^{2}}+y+2 \varepsilon y\left[1-5 \cos ^{2}\left(y^{2}+\left(\frac{a y}{d t}\right)^{2}\right)^{1 / 2} j=\epsilon^{2}\left(y^{2}+\left(\frac{d y}{d t}\right)^{2}\right\}^{1 / 2} \cos t\right.
$$

where $E$ \& 1 .

In the absence of the forcing function, this equation can be integrated exactly and exhibits the following behavior in the phase-piane of $y$ and $d y / d t$. Whenever the radius $r=\left[y^{2}+\dot{y}^{2}\right]^{1 / 2}$ in the phase-plane takes on the critical velues $r_{c}=\cos ^{-1}(5)^{-1 / 2}$, the motion reduces to simple harmonic asciliations with amplitude $r$ and unit frequency. For eech annular region bounded by two consecutive values of $F_{q}$, the interral curves are ovais with their axes aidigned alternately parailed either to $\mathrm{y}$ or to $\mathrm{dy} / \mathrm{dt}$. One would thus expect that the adaition of the forcing term with unit frequency will cause local resonance in neighoorhoods of the critical amplitudes $r_{c}$. As will be shown later on in this section, this vill indeed be the case and will give rise to the problem of the "critical amplitude".

Using the tro-variable method discussed by Cole and Kevorikinn (1962). (1963), the following form of the asymptotis expansion is first assuraed*

- Throuchout this paper the orission of the upper index on a summation symbol will indicate an asymptotic expansion. 
(2.2) $\quad y(t ; \varepsilon)=\sum_{i=0} y_{i}(t, \bar{t} ; \varepsilon) \varepsilon^{i}$

where the slow variuble $\bar{t}$ is derined by

(2.3)

$\overline{\mathrm{t}}=\varepsilon \mathrm{t}$

as discussec by Kevorkian (1902). Fnen the governini equation for $y_{0}$ is

(2.4) $\quad \frac{\partial^{2} y_{0}}{\partial t^{2}}+y_{0}=0$

whose general solution is

(2.5)

$$
y_{0}(t, \bar{t} ; \varepsilon)=a(\bar{t} ; \varepsilon) \cos [\bar{t}-B(t ; \varepsilon)]
$$

The functions $a(\bar{t} ; \varepsilon)$ and $B(\bar{t} ; \varepsilon)$ in $(2.5)$ which will be cailed "integration constents" will be determined by requiring $y_{2}$ to be bounded. For the present case ve al ways have the simfie harmonic operator on the left-hand side of all higher order equations. For simpiicity of calculations and for the explicit representation of the motion of the phase angle, we will expand the "integratior. constants" $a(\bar{t} ; \varepsilon)$ and $B(t ; \varepsilon)$ in the form:

$$
a(\bar{t} ; E)=\sum_{i=0} a_{i}(\bar{t})_{E}^{1} \quad B\left(\bar{t}_{; E}\right)=\sum_{i=0} B_{i}(\bar{t})_{E}
$$

From (2.1) the following equation for $g_{1}$ can be calculated:

$$
\frac{\partial^{2} y_{1}}{\partial t^{2}}+y_{1}=2 \frac{d a_{0}}{d \bar{t}} \sin (t-\beta)-2 \alpha_{0}\left[\frac{d \beta}{d \bar{t}}+\left(1-5 \cos ^{2} \alpha_{0}\right)\right] \cos (t-\beta)
$$

The boundedness of $y_{1}$ requires

$$
\text { (2.8) } \frac{d a_{0}}{d \bar{t}}=0 \quad \frac{d B_{0}}{d \bar{t}}=-\left(1-5 \cos ^{2} a_{0}\right) \equiv s_{0}
$$


These equations gire

(2.9)

$$
z_{0}=\text { const. } \quad s_{0}=s_{0} \bar{t}+b_{0}
$$

were $b_{0}$ is constant iepending on the initial condition. The solution for II is then

$(2.10)$

$$
J_{i}(t ; \vdots ; E)=0
$$

with no loss if Evaerality because the homogeneous solution is already accountea for in tise expasion of a and 3 in $y_{0}$;

Hor tine equation of $c\left(x^{2}\right)$ for $z_{2}$ is

$$
\begin{aligned}
& \frac{a^{2} y_{2}}{y^{2}}+y_{2}=\left[2 \frac{d}{d t}-a_{0} \sin B\right] \sin (t-B)+a_{0}\left[s_{0}^{2}-\frac{5}{2} \alpha_{0} s \sin 2 \alpha_{0}\right. \\
& \left.+\cos B+2 s a_{0}-2 \frac{d B}{d t}\right] \cos (t-B)-\frac{5}{2} \alpha_{0}^{2} s_{0} \sin 2 \alpha_{0} \cos 3(t-B)
\end{aligned}
$$

rhere

(2. 12)

$$
s_{0}^{\prime}=\frac{d s_{0}}{d a}=-5 \sin 2 a_{0}
$$

BJ the boundeciness requiremeat on $J_{2}$ we must set

(2.13e)

$$
\frac{a_{1}}{d \bar{t}}=\frac{a}{2} \sin
$$

$(2.130)$

$$
\frac{d z_{1}}{d \bar{t}}=\frac{s_{0}^{2}}{2}-\sum_{4} a_{0} s_{0} \sin 2 \alpha_{0}+\frac{1}{2} \cos B+s_{0} \alpha_{1}
$$

Since for $s_{0} \rightarrow 0$ (i.e. $\left.a_{0}=\cos ^{-1}(5)^{-1 / 2}\right)_{B}=b_{0}+O(\varepsilon)$, we see imediately from (2.13a) that $a_{2}$ becomes unbounded for large ralues of $i$. Thus, the expanaion proceaure assumed in (2.2) is not uniformly valid near the critical anplitudes. 
In this simpie moded the cause of the difficulty is casy to discern arn ronew. As was pointed out earlier, whenever $a=a_{c}=\cos ^{-1}(5)^{-1 / 2}$ the non-1inear srotem degenerates to simple harmonic motion with a frequency equal to that or the forcins function. Therefore, in soee neighborhood of $a_{c}$ the amilitüe must increase ippreciably before the non-iinear term comes into play and destroys the resonance of the forcing function. jue to this effect of iocal resonance the forcine, runction, wich woula otnervise be of order $\varepsilon^{2}$, now takes on a wore important role. This fact is exaiolted rathematicaliy in equations $(2.0)$. When $s_{0}$ is smail one cannot neelect tne nuener order iorciniz function in solving for $\beta_{b}$ and $a_{c}$. since in this case the rigit-hana sides of (2.8) are exclusively composed of smail terms. This fact was rirst pointed out by Struble (1961) in connection with the main problem.

In vieu of this, we anticipate the importance of the forcing functicn and introduce it immediately in the equations of order $z$. This means enuations (2.8) for a and $B$ nou become

$$
\frac{d a}{d \bar{t}}=\frac{c a}{2} \sin B \quad \frac{d B}{d t}=-\left(1-5 \cos ^{2} a\right)+\frac{\varepsilon}{2} \cos B
$$

The terms or order $E$ in (2.14), which are exclusively the contributions of the right-hand side of (2.1), will radically alter the behavior of and $B$ near the critical emplitudes.

Equations (2.14) are Hamiltonian, hence along an integral curve

(2.15) $2 \mathrm{H}=3 a+\frac{5}{2} \sin 2 a+\operatorname{cocos} \beta=$ const. 
With the aid of (2.15), the integral curves in the $a, 3$ plane can be easily calculated. The singuiar points are iocated at $b=B_{5}=n \pi, n=0, \pm 2, \pm 2, \ldots$ and $\cos \alpha=\cos \alpha_{s} \equiv \pm\left(1 / 5 \mp_{\varepsilon} / 10\right)^{1 / 2^{*}}$. These points form an alternating pattern of centers and saddle-points with solution curves as show qualitatively in Figure 1 .

We observe three possible types of notion if ve consider the integrai curves in vertical strips with a widh of order $\varepsilon^{1 / 2}$ centered about any of the critical amplitudes.

The integral curves which pass through two adjacent saddle-points for a given value of $a_{s}$ form the boundaries of oval resions with a width also of $O\left(\varepsilon^{1 / 2}\right)$ inside which both $\alpha$ and $B$ undergo bounded oscillations. For example the wotion in the neighborhood of the polnt $B=0$ and $a=a_{s}=\cos ^{-1}(1 / 5-\epsilon / 10)^{1 / 2}$ has the form

$$
\begin{aligned}
& a=\alpha_{s}+C_{1} E^{\dot{1 / 2}} \cos \left[\left(2 \varepsilon \alpha_{s}\right)^{1 / 2} \dot{\mathrm{t}}+\mathrm{C}_{2}\right] \\
& \text { (2.17) }=-4 c_{1}\left(2 a_{s}\right)^{-1 / 2} \sin \left[\left(2 c a_{s}\right)^{1 / 2} \dot{t}+c_{2}\right]
\end{aligned}
$$

where $c_{1}$ and $c_{2}$ are swall constants depending on initial conditions which allow us to linearize equations (2.14).

The separatrix forming the above boundary corresponds to motion where and $B$ approach the value at the saddle-point asymptotically as $\tilde{\mathrm{t}}+\cdots$. In fact, by use of (2.15) it is easy to show that the separatrix around the point $B=0$

- The upper or lover signs in the radical are to be taken when $B$ is an even or odd multiple of "respectively. 
and $a_{s}=\cos ^{-1}(1 / 5-\varepsilon / 10)^{1 / 2}$ for $0<a_{B}<\pi / 2$, intersects the $a$ axis at $a$ distance $(\varepsilon / 2)^{1 / 2} \cos ^{-1}(5)^{-1 / 2}+O(\varepsilon)$ from the gingular point. Finally, the wotion just outside the oscillatory regions is characterized by the fact that a undergoes bounded oscillations, wile $B$ has a secuiar motion superimposed on its oscillations. In all three of the above motions the characteristic frequency is $O\left(e^{3 / 2}\right.$ ) in the natural tine variable whereas the amplitudes of oscillation are $o\left(\varepsilon^{1 / 2}\right)(c f$. equations $(2.16)$ and $(2.17))$. This immediateiy suggests that the slov tine scale appropriate for motion near the critical amplitudes is $\bar{t}=\varepsilon^{3 / 2} t$, and that one must seek an expansion for $y$ in powers of $\varepsilon^{1 / 2}$.

As for the motion away from the critical anplitudes, we note from (2.8) and (2.13) that a oscillates with amplitude and frequency of order $\varepsilon$, and that the osclllatory as well as secular components of $B$ behave similariy.

The above intuitive construction vill next be analyzed systematically by the use of two aifferent expansions and their roles established in teras of all possible initial conditions.

\subsection{Outer expansion}

In order to account for the most general form of initial conditions, ve represent the motion aray from the critical amplitude by an expansion in porers of $\varepsilon^{1 / 2}$, called the outer expansion:

$$
y(t ; \varepsilon)=\sum_{i=0} y_{i / 2}(t, \bar{t} ; \varepsilon)^{1 / 2}
$$




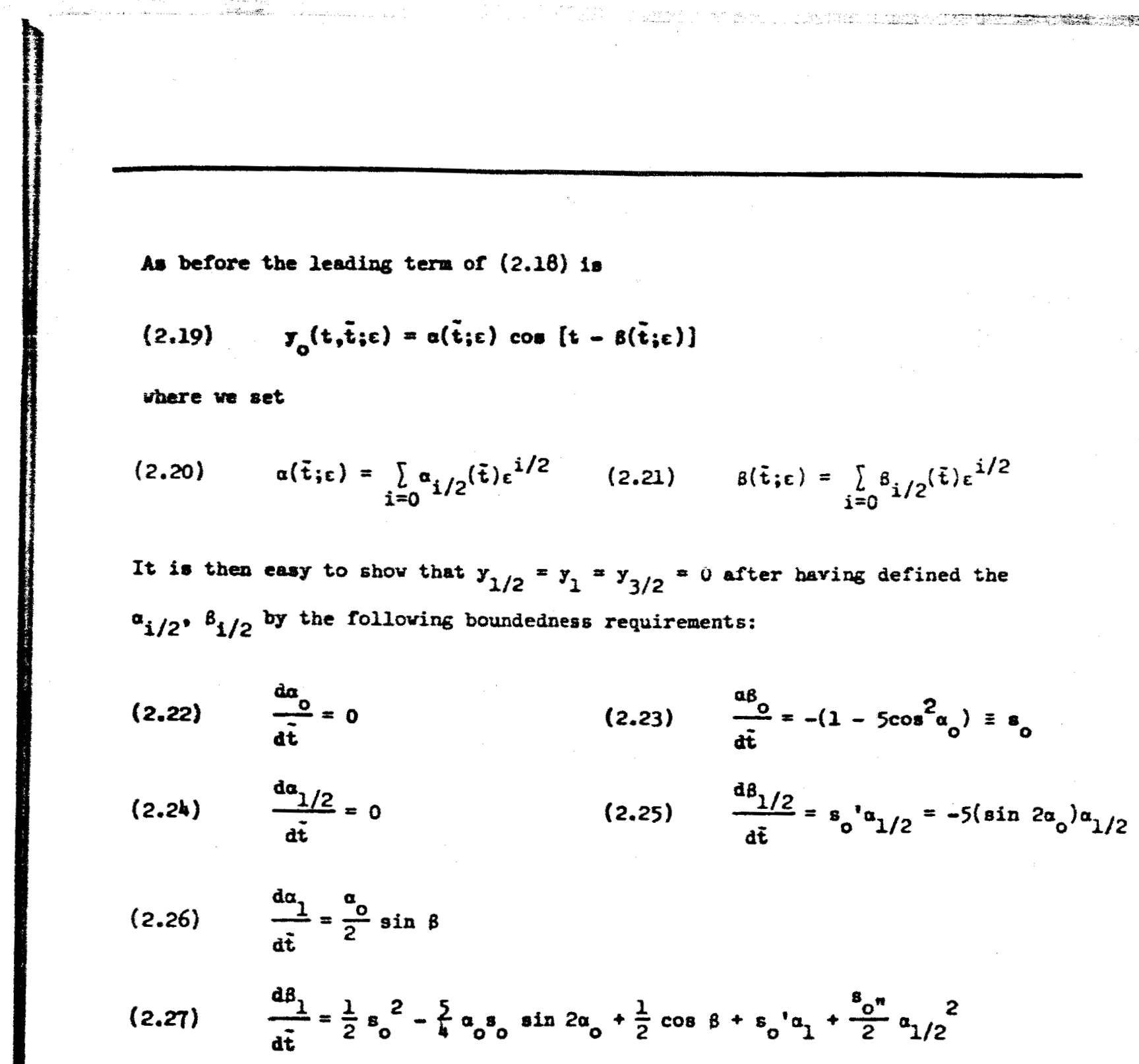

were

$$
a_{0}=\frac{d^{2} s_{0}}{d \sigma_{0}^{2}}=-10 \cos 2 a_{0}
$$

Wote that trigonometric functions with $B$ as argument are not expanded to aroid trivial non-uniformities as the expansion of $\beta$ in (2.21) need not involve bounded functions. It is only the phase relocity $\mathrm{d} \beta / \mathrm{d} \dot{\mathrm{t}}$ thet must be bounded. 
The solutions of the above equations are:

(2.28)

$$
a_{0}=\text { const. }=a_{0}
$$

(2.29) $\quad B_{0}=\varepsilon_{0} \bar{z}+b_{0}$

and

12.30

$$
a_{1 / 2}=\text { const. }=a_{1 / 2}
$$

$$
B_{1 / 2}=s_{0}^{1} a_{1 / 2} \bar{t}+b_{1 / 2}
$$

anà

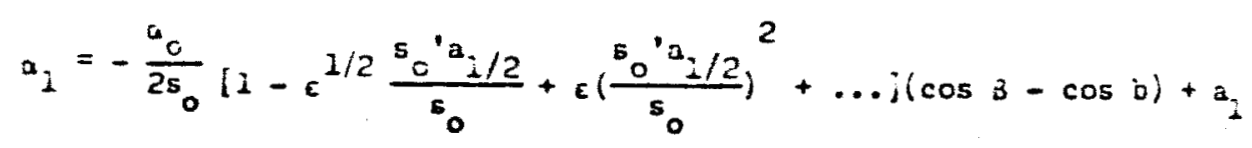

and equation $(2.27)$ reduces to

$$
\begin{aligned}
& \frac{a B_{1}}{d \bar{t}}=\frac{2}{2} s_{0}^{2}-\frac{5}{4} \alpha_{0} s_{0} \sin 2 a_{0}+\frac{2}{2} \cos B-\frac{s_{0} \alpha_{0}}{2 s_{0}}\left[1-\varepsilon^{1 / 2} \frac{s_{0}{ }^{B_{1}} 1 / 2}{s_{0}}\right. \\
& +c\left(\frac{s_{0}^{\prime} a_{1 / 2}}{s_{0}}\right)^{2}+\ldots l(\cos B-\cos b)+s_{0}^{\prime} a_{2}+\frac{s_{0}^{\prime \prime}}{2} a_{1 / 2} 2
\end{aligned}
$$

if the initial conditions are given as

$$
\begin{aligned}
& 3=b=b_{0}+\varepsilon^{1 / 2} b_{1 / 2}+\varepsilon b_{1}+\ldots \\
& a=a=a_{0}+\varepsilon^{1 / 2} a_{1 / 2}+\varepsilon a_{1}+\ldots
\end{aligned}
$$

et $\mathrm{t}=0$.

Equation (2.32) for $a_{1}$ exhibits the non-uniformity of the expension near $s_{0}=0$. Wote that $a_{1 / 2}$ would be identicaliy zero if the initial amplitude did not contain a term proportional to $\varepsilon^{1 / 2}$. 


\subsection{Inner expansion}

An wentioned preriously, the outer expansion fails to be valid as $s_{0} \rightarrow 0$. He now seek a salution which is ralid and does not becone unbounded at the critical amplitudes. This expansion vill be called the "inner expansion". We let (cr. discussion after Fig. 1)

$$
\text { (2.35) } \quad s_{0}=\varepsilon^{1 / 2} \bar{s}_{0}
$$

and assume the following expansion for $y$

$$
y(t ; \varepsilon)=\sum_{i=0} y_{i / 2}\left(t, \bar{t}_{j \varepsilon}\right)_{\varepsilon}^{i / 2}
$$

where a new slow rariable

$$
\bar{t}=\varepsilon^{3 / 2} t=\varepsilon^{i / 2} \bar{t}
$$

has been chosen. The equation for $y_{0}$ is again

(2.38) $\quad \frac{\partial^{2} y_{0}}{\partial t^{2}}+y_{0}^{*}=0$

vhose general solution can be written in the form:

(2.39)

$$
Y_{0}^{*}(t, \bar{t} ; \varepsilon)=Q^{*}(\bar{t} ; \varepsilon) \cos \left[t-B^{*}\left(\bar{t}_{;} ; \varepsilon\right)\right]
$$

We also expand the slowly varying functions $a(\bar{t} ; \varepsilon)$ and $B(\bar{t} ; \varepsilon)$ in the following

form in order to account for the homogeneous solutions of all higher orders.

$$
a^{*}(\bar{t} ; \varepsilon)=\sum_{i=0} a_{i / 2}(\bar{t}) \varepsilon^{i / 2} \quad B\left(\bar{t}_{i \varepsilon}\right)=\sum_{i=0} B_{i / 2}(\bar{t}) \varepsilon^{i / 2}
$$


Substitution of the above expansions into (2.1) and the requirement that the $y_{i / 2}$ be bounded gives the following ordinary differential equations for the $a_{1 / 2}$ and $s_{1 / 2}:$

(2.41) $\frac{d e_{0}}{d \bar{t}}=0$ $\frac{d B_{0}}{d \bar{t}}=\varepsilon_{0} \alpha_{1 / 2}$

$(2.42 a) \quad \frac{d a_{1 / 2}}{d \bar{t}}=\frac{a_{0}}{2} \sin b^{*}$

$$
\frac{d B_{1 / 2}}{d \bar{t}}=s_{0}{ }^{\prime} \alpha_{1}+\frac{s_{0}^{\prime}}{2}\left(a_{1 / 2}\right)^{2}+\frac{1}{2} \cos B^{\prime}
$$

$(2.43 a) \quad \frac{d a_{1}}{d t}=\frac{a_{1 / 2}}{2} \sin B$

$(2.43 b) \quad \frac{d B_{1}}{d \bar{t}} s_{0}{ }^{\prime} a_{3 / 2}+s_{0}{ }^{\prime a} a_{1 / 2} a_{1}+\frac{\delta_{0}^{\prime \prime \prime}}{6}\left(a_{1 / 2}\right)^{3}-\frac{5}{2} a_{0} \bar{s}_{0} \sin 2 a_{0}$

vith the additional results that

$$
y_{1 / 2}^{*}=y_{1}^{*}=t_{3 / 2}=y_{2}^{*}=0
$$

and that only in $y_{5 / 2}$ do we have higher harmonics in the fast variable.

We note that equations $(2.42)$ are precisely the equations one vould obtain in the inner limit from (2.14). Equations. (2.41-2.43) can be solved successively for the $a_{1 / 2}$ and the results are summarlized below.

$$
\begin{array}{ll}
(2.45 a) & a_{0}^{\prime}=\text { const. }=a_{0}^{*} \\
(2.45 b) & a_{1 / 2}=\bar{K}_{0}+\frac{1}{s_{0}}\left[\bar{K}_{1}^{2}+\bar{K}_{2}\left(\cos B^{*}-\cos b^{*}\right)\right]^{1 / 2} \\
(2.45 c) & a_{1}=-\frac{\bar{K}_{0}}{\bar{K}_{2}}\left[\bar{K}_{1}^{2}+\overline{\mathrm{K}}_{2}\left(\cos B^{*}-\cos b^{*}\right)\right]^{1 / 2}-\left(\cos B^{*}-\cos b^{*}\right) / 2 s_{0}^{\prime}+a_{1}^{*}
\end{array}
$$


iㅏ를

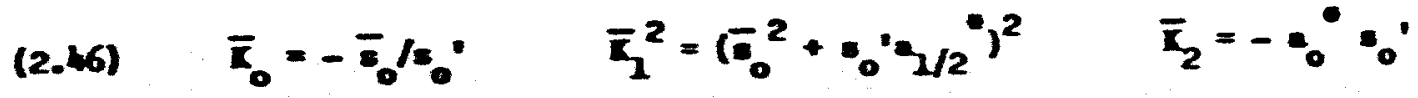

and the initial conditions ot $\bar{t}=0$

$$
\text { (2.h7) } \quad v_{i / 2}=a_{i / 2}=b
$$

bare beed inposed.

Wth the $a_{i / 2}$ - so derined the nalution for the $b_{i / 2}$ reduces to quadratures. Theae detalls $\mathrm{hll}$ wot be carried ont bere $s$ the qualitative beharior of both a and 8 hare aread been discused. 
2.4 Matching of solutions and composite expansion

In the standard singular perturbation problem in which two linit process expansions can be derived in their respective domains, either one or both of these expansions is defined incompletely prior to the natching (cr. Kaplun and Lagerstrom (1957)). For example, the initial conditions for the inner solution would depend upon time values taken on by the outer solution in the inner region if the motion spans both regimes (cf. Lagerstrom and Kerorikian (1963)). In this case, the matching will define the motion in the inner region and the beharior of the two limit-process expansions in their comon overlap domain wll provide the basis for deriving a composite expansion which is untromily valid everywhere.

In the present example, as vell as in the main problell, the motion depending upon the initial condition on a lies for all times in either the outer or inner regions. Furthermore, the parameter which establishes the appropriate expansion does not vary in order of agnitude vith time. The purpose of matching is then two-fold. First, the direct matching of the two expansions v1l prove the existence of a comon overiap domain and rule out the possibility of an even third limit-process expansion for some value of such that $\delta_{0}=\left(\varepsilon^{\prime \prime}\right), 1<\mu<1 / 2$. Secondly, the watching vill provide the necessary Information for obtaining a representation of the motion for all ralues of - In the above order interval once the behaviors at the end-points of this order interval have been calculated. General principles of matching are discussed by Kaplun and Lagerstrom (1957). For the present examples, as vell as for the main problem, it is sufficient to show that the inner solution for large values of $\overline{8}_{0}$ agrees with the inner limit of the outer expansion. In 
this ereat the derivation of composite expansion which is uniforaly vaid for all $s_{0}$ in the order interval ord $\varepsilon^{1 / 2} \leq$ ord $s_{0} \leq$ ord 1 becomes particuiarij etraightrorvard.

The matching between $a$ and $a$ is rery simple. If ve rewrite $a$ in terms of outer variables and expand for $\vec{s}_{0}+\cdots$, ve obtain

$$
\begin{aligned}
& a=a_{0}+\varepsilon^{1 / 2} a_{1 / 2}+c_{1}+o\left(c^{3 / 2}\right)=a_{0}+\varepsilon^{1 / 2} a_{1 / 2}+c_{1} \\
& -\frac{c}{2} \frac{a_{0}}{B_{0}}\left(\cos B^{*}-\cos b^{\circ}\right)+\frac{1}{2} \varepsilon^{3 / 2}\left(\frac{s_{0}}{s_{0}} a_{0}^{-}-1\right) a_{1 / 2}\left(\cos b^{*}-\cos b^{*}\right) \\
& -\frac{c^{2}}{2}\left(\frac{s_{0}^{\prime}}{s_{0}} a_{0}^{*}-1\right)\left[\frac{s_{0}^{\prime}}{s_{0}^{2}} a_{1 / 2}\left(\cos B^{*}-\cos b\right)+\frac{1}{4} \frac{a_{0}}{s_{0}^{2}}\left(\cos ^{2} b\right.\right. \\
& \left.\left.-2 \cos B^{\circ} \cos b^{\circ}+\frac{1}{2}+\frac{1}{2} \cos 2 B^{\circ}\right)\right]+O\left(\varepsilon^{5 i 2}\right)
\end{aligned}
$$

Frow the outer expansion we have

$$
\begin{aligned}
& a=a_{0}+c^{1 / 2} a_{1 / 2}+c a_{1}+0\left(c^{3 / 2}\right)=a_{0}+c^{1 / 2} a_{1 / 2}+c a_{1} \\
& -\frac{c a_{0}}{2 s_{0}}\left[1-\varepsilon^{1 / 2} \frac{s_{0}^{1 a_{1}} 1 / 2}{s_{0}}+\varepsilon \frac{0_{0}^{2} a_{1 / 2}}{s_{0}^{2}}+\ldots\right](\cos B-\cos b)+0\left(c^{5 / 2}\right)
\end{aligned}
$$

By comparing equations (2.48) and (2.49) we see that the inner expansion contains the outer expansion explicitly to order $\varepsilon^{2}$. Note that in the orerlap dowain we have $a_{i / 2}=a_{i / 2}$ - In fact, all terms in the outer expansion to order $\varepsilon^{2}$ are contained in $a_{0}+\epsilon^{1 / 2} a_{1 / 2} \cdot$. The outer expansion of $a_{1}$ is entirely or higher order. Thus, the composite expangion which is uniformly ralid to $O(c)$ everywhere is:

$$
a_{c}=a_{0}^{0}+c^{1 / 2} a_{1 / 2}+c a_{1}
$$


In this matching, we have assumed that both $B$ and $\beta$ are matchec. Fis $-i 2 i$ be shown in the subsequent discussion. For simplicity, ve will discuss the matching between $\mathrm{d} s / \mathrm{d} \overline{\mathrm{t}}$ and $\mathrm{d} \bar{s}^{*} / \mathrm{d} \bar{t}$ instead.

To sumarize, we have already obtained

$$
\begin{aligned}
& \frac{d B}{d \tilde{t}}=s_{0}+\varepsilon^{1 / 2} s_{0} a_{1 / 2}+\varepsilon\left(\frac{1}{2} s_{0}^{2}-\frac{5}{4} a_{0}^{2} s_{0} \sin 2 \alpha_{0}\right. \\
& \left.+\frac{i}{2} \cos B+s_{0} a_{1}+\frac{s_{0}^{\pi}}{2} a_{1 / 2}{ }^{2}\right)+o\left(E^{3 / 2}\right)
\end{aligned}
$$

and

$$
\frac{a 3}{d \bar{t}}=\bar{s}_{0}+s_{0}{ }^{\prime} \alpha_{1 / 2}=\varepsilon^{1 / 2}\left[s_{0}{ }^{\prime} \alpha_{1}^{*}+\frac{i}{2} \cos B+\frac{s_{0}^{\prime \prime}}{2}\left(\alpha_{1 / 2}\right)^{2}\right]+o(\varepsilon)
$$

We note that the inner expansion of (2.52) for dB/at contains all the terms that appear in the outer expansion (2.51) with the exception of the two terms

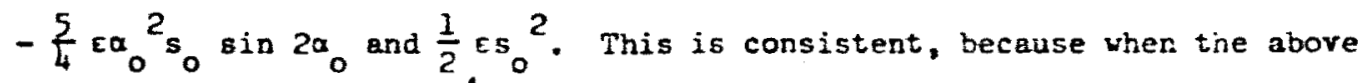
terms are expressed in terms of the inner parameter $\bar{s}_{0}$, they become of oroer $\varepsilon^{3 / 2}$ and $\varepsilon^{2}$ respectively. Thus, they should sppear in the expressions for $\mathrm{dB}_{1} / \mathrm{dt}$ and $\mathrm{dB} \mathrm{B}_{3 / 2} / \mathrm{dt}$ respectively. The first term does appear in the expression (2.43b) for $d B_{1}{ }^{\bullet} / d \bar{t}$ and one vould recover the second term if $\mathrm{dB}_{3 / 2} / \mathrm{d} \overline{\mathrm{t}}$ vere evaluated.

Conversely, many terms in the inner expansion, e.z. $5_{0}{ }^{\prime} a_{1}$ " and $s_{0}{ }^{n}\left(a_{1 / 2}{ }^{*}{ }^{2} / 2\right.$, are of orders higher than we considered in the outer expansion and vil2 apt zar in the corresponding higher order terms. Having carried out the calculations to the present order we can easily derive the following composite expansion for $d B_{c} / d \bar{t}$ which is uniformly valid to order $\varepsilon^{2}$ for $a 11 \mathrm{~s}_{0}$. 
(2.53)

$$
\begin{aligned}
& \frac{d \bar{s} c}{d \bar{t}}=\bar{s}_{0}+s_{0} a_{1 / 2}+\varepsilon^{1 / 2}\left[s_{0} a_{1}+\frac{1}{2} \cos b^{*}+\frac{s_{0}}{2}\left(a_{1 / 2}\right)^{2} ;\right. \\
& -\sum_{b} c\left(a_{0}\right)^{2} \bar{s}_{0} \sin 2 a_{0}+\frac{c^{2}}{2} \bar{s}_{0}^{2}
\end{aligned}
$$

In deriving (2.53) we have used tine custowary construction of adaing tise injer and outer representations for $d B / d t$ and subtracting those teras vinicin are comon to both expansions in the intermediate refion. These teras are tie two higher order terms appearing at the end of (2.53). Thus, to oraer $c^{1 / 2}$ the Inner expansion $d B^{*} / \mathrm{dt}^{-}$is itself uniformly valia for all $\mathrm{s}_{0}$. It is only in deriving an expression valid to orders higher than $c^{1 / 2}$ that one needs consideration of terms contributed by the outer expansion.

Finally, the solution of (2.1) for $y$ which is uniformly valid to o(e) for 211 s is

$$
y(t, c)=\left(a_{0}+\varepsilon^{1 / 2} a_{1 / 2}+c_{2}\right) \cos \left[t-\beta_{c}(t ; \varepsilon)\right]+0\left(\varepsilon^{3 / 2} j\right.
$$

The behavior of the amplitude and phase to $O\left(\varepsilon^{1 / 2}\right)$ was aiscussed eariier in connectlon with $(2.14)$. The higher order terms vill not alter the generai qualitative nature of the solution. The detailed and systenatic develozent of the expansions for $a$ and $B$ was carried out here to serve as a guideiine for the study of the main problem for which there is no a priori knowled of tice particular higher order terms which cause local resonance. Hence cne mist rely on a more formal construction analagous to the process used in sections $2.2-2.4$ 


\section{The ilain Probies}

Once suitable choice of variables is made, the motion of a sateilite aroma as oblate pianet reduces in principle to the solution of a problea in non-linear oscillations analogous to the model discussea in section 2. Or conrse, instead of the two siosily varyinf functions $\alpha$ and $B$, Je now bare six siovis varyine orbitai elements. Hovever, it will be shorn tinat the sain probie hinges on solving the coupled equations for the inclination and apse vinieh will be the analogues of a and $B$, and that the remainder of the eienents vill then be given by quadratures.

\subsection{Formulation of the problem, coordinate systen}

Consider an inertial frame vith origin at the center of an oblate pianet haring a radius $R$ in the equatorial plane of symetry. Te noraalize jistances by the radius $R$ and the time oy $\left(R^{3} / G 4\right)^{1 / 2}$, where $G$ is the nniversal graritational constant and $M$ is the mass of the planet. The dimensionless equation of motion for a satelite is then

$$
\frac{d^{2} x}{a t^{2}}=\operatorname{grad} U
$$

were $\vec{x}$ is the dimensionless distance vector from the origin and the potential $U$ has the following form in spherical polar coordinates with respect to the polar axis of symetry:

$$
U=\frac{1}{r}+\frac{\varepsilon}{3 r^{3}}\left(1-3 \cos ^{2} \theta\right)+\frac{c \varepsilon^{2}}{5 r^{5}}\left(35 \cos ^{4} \theta-30 \cos ^{2} \theta+3\right)+o\left(\varepsilon^{3}\right)
$$

where $\theta$ is the polar angle. 
It has been assumed that the planet is an elipboid of revolution ana for the earth the constants $c$ and $c$ are approximately (cr. Jeffries (1959) and Shi (1963))

$$
E=I=1.623 \times 10^{-3} \quad c=4 / 7
$$

In the conventional spherical polar coordinates:

(3.3a) $\quad x=r \cos \psi \sin \theta$

(3.3b) $\quad y=r \sin \sin \theta$

(3.3c) $z=r \cos \theta$

where

(3.3d) $\quad \vec{x}=(x, y, z),|\vec{x}|=r$

Equation (3.1) for any potential $U$ has the following component form:

(3.4a) $\frac{d}{d t}\left(r^{2} \sin ^{2} \theta \frac{d \phi}{d t}\right)=\frac{\partial U}{\partial \psi}$

(3.4b) $\frac{d}{d t}\left(r^{2} \frac{d \theta}{d t}\right)-r^{2} \sin \theta \cos \theta\left(\frac{d \phi}{d t}\right)^{2}=\frac{\partial U}{\partial \theta}$

(3.hc) $\frac{d^{2} r}{d t^{2}}-r\left(\frac{d \theta}{d t}\right)^{2}-r \sin ^{2} \theta\left(\frac{d \dot{d}}{d t}\right)^{2}=\frac{\partial U}{\partial r}$

Since the satellite can be considered to move in an instantaneous plane defined by the distance and relocity rectors, one may also define the motion by the fallowing variables proposed by Struble (1960) and (1961) (cr. Fig. 2 for the geonetryl.

1 = angle betreen instantaneous orbital and equatorial planes

$Q=$ angle in the equatorial plane between some fixed direction, say $x$ pointing tovards the rernal equinox, and the ascendins node

$\mathbf{r}=$ the radius

- angle between the ascending node and the distance rector. 
Struble (1960) has shown that equations (3.4) transform to the followirs fifth-order system after elimination of the time."

$$
\begin{aligned}
& \frac{d n}{d \phi}=\frac{\frac{\partial U}{\partial \dot{\theta}}}{\frac{m^{2}}{\cos i} \cdot \frac{\cos ^{3} i \cos \theta}{p \sin ^{2} i \sin 6}\left[\frac{\partial U}{\partial \theta}+\tan i \frac{\cos \theta}{\sin \frac{\partial U}{\partial \varphi}}\right]} \\
& \frac{\partial s_{l}}{d \theta}=\frac{-\cos ^{3} i \cos 6\left[\frac{\partial j}{\partial j}+\tan i \frac{\cos \theta}{\sin \theta} \frac{\partial U}{\partial y}\right]}{p^{2} u^{2} \sin ^{2} i \sin \theta+\cos ^{4} i \cos \theta\left[\frac{\partial j}{\partial \partial}+\tan i \frac{\left.\cos \frac{\partial}{\sin \theta} \frac{\partial U}{\partial \psi}\right]}{\sin }\right.}
\end{aligned}
$$

$$
\frac{d i}{d \phi}=-\frac{\sin ^{2} i \cos ^{3} i \cos \phi\left[\frac{\partial U}{\partial \theta}+\tan i \frac{\cos \theta}{\sin \theta} \frac{\partial U}{\partial \dot{y}}\right]}{p^{2} u^{2} \sin ^{2} i \sin \theta+\cos i \cos \theta\left[\frac{\partial U}{\partial \theta}+\tan i \frac{\cos \phi}{\sin \theta} \frac{\partial U}{\partial \phi}\right]}
$$

$$
\frac{d^{2} u}{d \phi^{2}}-\frac{2}{u}\left(\frac{d u}{d \phi}\right)^{2}+\frac{\frac{d u}{d \phi} \cdot \frac{d}{d \phi}\left(\frac{d \phi}{d t}\right)}{\frac{d \phi}{d t}}=-\frac{\frac{p^{2} u^{5}}{\cos ^{2} i}+u^{2} \frac{\partial U}{\partial r}}{\left(\frac{d \phi}{d t}\right)^{2}}
$$

where $p$ is the component of angular mosentum aions the polar axis and is defined by

$$
\begin{array}{ll}
(3.5 e) & p=r^{2} \sin ^{2} \frac{d \phi}{d t} \\
(3.5 r) & u=\frac{1}{r}
\end{array}
$$

In equation (3.5d) $\frac{\mathrm{d}}{\mathrm{dt}}$ and $\theta$ are derined by

$$
\frac{d \phi}{d t}=\frac{p u^{2}}{\cos i}+\frac{\cos ^{3} i \cos \theta}{p \sin ^{2} i \sin \theta}\left[\frac{\partial U}{\partial \theta}+\tan i \frac{\cos \theta}{\sin } \frac{\partial U}{\partial v}\right]
$$

- Wote that Struble (1960) defines the node in the opposite sense. 
(3.5h)

If we now use (3.2) for $U$ and retain teras up to $O\left(\varepsilon^{2}\right)$ only, (3.5) simplify to (3.6e) $\frac{d p}{d s}=0$

$$
\frac{d \Omega}{d \phi}=\frac{-2 r u \cos ^{3} i \cos ^{2} \theta\left(2-2 \operatorname{ces} u^{2}\left(7 \cos ^{2} \theta-3\right)\right]}{p^{2} \sin ^{2} i+2 \varepsilon u \cos ^{4} i \cos ^{2} \theta\left[1-2 \operatorname{ceu} u^{2}\left(7 \cos ^{2} \theta-3\right)\right]}
$$

$$
\frac{d i}{d \theta}=\frac{-2 \varepsilon u \sin ^{2} i \cos ^{3} i \cos \theta \cos \theta\left[2-2 \operatorname{cen}{ }^{2}\left(7 \cos ^{2} \theta-3\right)\right]}{p^{2} \sin ^{2} i+2 \varepsilon u \cos ^{4} i \cos ^{2} \theta\left[1-2 \operatorname{ceu} u^{2}\left(7 \cos ^{2} \theta-3\right)\right]}
$$

$$
\begin{aligned}
& \frac{d^{2} u}{d \phi^{2}}-\frac{2}{u}\left(\frac{d u}{d \phi}\right)^{2}+\frac{\frac{d u}{d \phi} \cdot \frac{d}{d \phi}\left(\frac{d \phi}{d t}\right)}{\frac{d \phi}{d t}}=-\frac{p^{2} u^{5}}{\left(\frac{d \phi}{d t}\right)^{2} \cos ^{2} i} \\
& +\frac{1+\operatorname{cu}^{2}\left(1-3 \cos ^{2} \theta\right)+c \varepsilon^{2} u^{4}\left(35 \cos ^{4} \theta-30 \cos ^{2} \theta+3\right)}{\left(\frac{d \phi}{d t}\right)^{2}} \cdot u^{4}
\end{aligned}
$$

where $\frac{d \phi}{d t}$ is given by $(3.5 E)$ vith $\frac{\partial U}{\partial y}=0$.

According to $(3.6 a) p$ is a constant, a consequence of the independence of $y$

on *. Furthermore, equation (3.6b) for the node is uncoupled fron (3.6c) and (3.6d) and can hence be soived independentiy once $u$ and $i$ have been determined.

Making use of the identities $(3.58)$ and $(3.5 \mathrm{~h})$ and retaining terms up to $O\left(c^{2}\right)$ in $(3.6 c)$ and $(3.6 d)$ yields:

$$
\begin{aligned}
& \frac{d i}{d \phi}=-\frac{e}{p^{2}} \cos ^{3} i \sin i \sin 2 \phi+2 \varepsilon^{2} \frac{u^{2}}{p^{2}} \cos ^{3} i \sin i\left[\frac{l}{p^{2}} \cos ^{4} i \sin ^{2} \phi\right. \\
& \left.-3 c u+7 c u \sin ^{2} i \sin ^{2} \phi\right] \sin 2 \phi+o\left(\varepsilon^{3}\right)
\end{aligned}
$$


$(3.7 b)$

$$
\begin{aligned}
& \frac{d^{2} u}{d \phi^{2}}+u=\frac{\cos ^{2} i}{p^{2}}+c\left[\frac{4 u^{2}}{p^{2}} \cos ^{4} i \sin ^{2} \phi+\frac{u}{p^{2}}\left(\frac{d u}{d \phi}\right) \cos ^{2} i\left(1-3 \cos ^{2} i\right) \sin 2 \phi\right. \\
& \left.-\frac{2}{p^{2}}\left(\frac{d u}{d \phi}\right)^{2} \cos ^{4} i \sin ^{2} \phi+\frac{u^{2}}{p^{2}} \cos ^{2} i\left(1-3 \sin ^{2} i \sin ^{2} \phi\right)-4 \frac{u}{p^{4}} \cos ^{6} i \sin ^{2} \phi\right] \\
& +\varepsilon^{2}\left[-4 \frac{\cos ^{4} i}{p^{2}} u^{3} \sin ^{2} \phi i \frac{3 \cos ^{4} i}{p^{2}} \sin ^{2} \phi-2 u c\left(3-7 \sin ^{2} i \sin ^{2} \phi\right)\right\} \\
& -8 \frac{u^{2}}{p^{4}} \frac{d u}{d \phi} \sin ^{2} i \cos ^{6} i \sin ^{2}+\operatorname{in} 2-2 c \frac{u^{3}}{p^{2}} \frac{d u}{d \phi} i-3 \sin ^{2} i \cos ^{2} i \\
& \left.+7 \sin ^{4} i \cos ^{2} i \sin ^{2} \phi+6 \cos ^{4} i-28 \cos ^{4} i \sin ^{2} i \sin ^{2} \phi\right\} \sin 2 \phi \\
& -12 c \frac{u^{2}}{p^{2}}\left(\frac{d u}{d \phi}\right)^{2} \cos ^{4} i \sin ^{2} \phi\left(3-7 \sin ^{2} i \sin ^{2} \phi\right) \\
& +2 \frac{u^{2}}{p} \frac{d u}{d \phi} \cos ^{6} i\left(3 \cos ^{2} i-1\right) \sin ^{2} \phi \sin 2 \phi+4 \frac{u}{p}\left(\frac{d u}{d \phi}\right)^{2} \cos ^{8} i \sin ^{4} \phi \\
& +c \frac{u^{4}}{p^{2}} \cos ^{2} i\left\{35 \sin ^{4} i \sin ^{4}-30 \sin ^{2} i \sin ^{2} \phi+3\right\} \\
& -4 \frac{u^{3}}{p^{4}} \cos ^{6} i \sin ^{2}+\left(1-3 \sin ^{2} i \sin ^{2} \phi\right)+4 \frac{u^{2}}{p^{4}} \cos ^{6} i \sin ^{2} \phi\left(\frac{3}{p^{2}} \cos ^{4} i \sin ^{2} \phi\right. \\
& \left.\left.-2 \operatorname{cu}\left(3-7 \sin ^{2} i \sin ^{2} \phi\right)\right\}\right]+o\left(\varepsilon^{3}\right)
\end{aligned}
$$

It is mentioned in passing that struble (1961) chose a modified rariable analogous to in order to eliminate certain non-uniformities in the solution. With the present approach this is unnecessary, since all the required scale changes are automatically accounted for by the two-rariable procedure. 


\subsection{Outer exgension}

The ain proble to which ve bave previously referred is the solution of equations (3.7n) and (3.ib). Since $\cos ^{2} i / p^{2}$ is constant to orier unity, ve see from (3.Tb) that this problen reduces to solving the motion of a oseillator vith anall non-linearities and a weak coupling because $i$ is constant to onder unity. The socerisat iengtig nature of the perturbation serns in (3.To) cces not alter tine fuct inat ice systen in question is qualitativeig acalosous to the model equation stuicied is aecion 2. We therefore proceed as in Section 2.2 by assuning the following expansions for $i$ and $u$ :

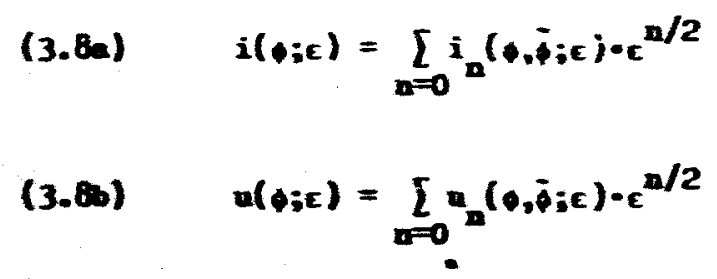

where analogous to the sion time rariable, is defined by

$$
\text { (3.6e) } \bar{\phi}=\mathrm{c}
$$

Substitution of (3.8) into (3.7) gives to order unity

$$
\begin{aligned}
& \text { (3.9a) } \frac{\partial i_{0}}{\partial \phi}=0 \\
& \text { (3.90) } \frac{\partial^{2} u_{0}}{2 p^{2}}+u_{0}=\frac{\cos ^{2} i_{0}}{p^{2}}
\end{aligned}
$$

whose general solution is

$$
\begin{aligned}
& \text { (3.10a) } \quad i_{0}=i_{0}(i ; \varepsilon) \\
& (3.10 b) \quad p_{0}=\frac{\cos ^{2} i_{0}}{p^{2}}(i+e(t ; \varepsilon) \cos [\phi-\psi(i ; \varepsilon)])
\end{aligned}
$$


In $(3.10 \mathrm{~b})$ the two "constants of integration" have been expressed in terms of the conventional Keplerian elements.
e eccentricity
$\omega$ = apse angle measured in the counterclockribe sense from the ascendins node to perigee in the instantaneous orbital plane.

As before ve assime $i_{0}, e$ and whave the following expressions

$$
\begin{aligned}
& (3.11 \varepsilon) \quad i_{0}(j ; \varepsilon)=\sum_{n=0} i_{\text {on }} / 2^{(\bar{\phi}) \varepsilon^{n / 2}} \\
& \text { (3.11b) e(j;c) }=\sum_{n=0} e_{n / 2}(\bar{\phi}) e^{n / 2} \\
& \text { (3.11c) } \quad \omega(\bar{\phi} ; \varepsilon)=\sum_{n=0} n / 2(\bar{\phi}) e^{n / 2}
\end{aligned}
$$

in order to account for the homogeneous solutions of the higher order terms in $i$ and $u$. It is easy to see that since terms of $o\left(\varepsilon^{1 / 2}\right)$ are absent in $(3.7)$, $i_{1 / 2}=u_{1 / 2}=0$. The following equations for $i_{1}$ and $u_{1}$ can then be derived.

(3.12a) $\frac{\partial i_{1}}{\partial \phi}=\frac{d i}{\partial i}-\frac{1}{p^{4}} \cos ^{5} 1_{00} \operatorname{\theta ini} 00 \sin 2 \phi\left[1-e_{0} \cos (\phi-\omega)\right]$

$(3.12 b) \quad \frac{\partial^{2} u_{1}}{2 \phi^{2}}+u_{1}=\left[-\frac{2 e_{0}}{p^{2}} \frac{d \omega}{d i} \cos ^{2} i_{\infty}-\frac{e_{0}}{p^{6}} \cos ^{6} i_{\infty}\left(1-5 \cos ^{2} i_{\infty}\right)\right] \cos (\omega-\omega)$

$$
\begin{aligned}
& +\frac{2}{p^{2}} \cos ^{2} i_{00} \frac{d e_{0}}{d \phi} \sin (\phi-\omega)-\frac{1}{p^{6}} \cos ^{6} i_{\infty} \sin ^{2} i_{\infty}\left[\cos 2 \phi+\frac{e_{0}}{3} \cos (3 \phi-\omega)\right] \\
& +\frac{\cos ^{6} i_{\infty}}{p^{6}}\left(-\frac{1}{2}+\frac{1}{2} \cos ^{2} i_{\infty}\right)\left[1+\frac{e_{0}^{2}}{2}+\frac{e_{0}^{2}}{2} \cos 2(\phi-\omega)\right] \\
& +\frac{\cos ^{6} i_{00}}{2^{6}}\left(3-7 \cos ^{2} i_{\infty}\right)\left[\left(1+\frac{e_{0}^{2}}{2}\right) \cos 20+e_{0} \cos (3 \phi-\omega)\right.
\end{aligned}
$$




$$
\begin{aligned}
& \left.+\frac{e_{0}^{2}}{4} \cos (4 \phi-2 \omega)+\frac{e_{0}^{2}}{4} \cos 2 \omega\right]-\frac{2}{p^{6}} \cos ^{8} i_{00}\left[1-\cos 2 \phi-\frac{e_{0}}{2} \cos (3 \phi-\omega)\right] \\
& -\frac{e_{0}}{2} \frac{\cos ^{6} i_{\infty}}{p^{6}}\left(1-3 \cos ^{2} i_{\infty}\right)\left[-\cos (3 \phi-\omega)-\frac{e_{0}}{2} \cos (4 \phi-2 \omega)+\frac{e_{0}}{2} \cos 2 \omega\right) ! \\
& -\frac{e_{0}^{2}}{2} \frac{\cos ^{8} i_{\infty}}{p^{6}}\left[1-\cos 2(\phi-\omega)-\cos 2 \phi+\frac{1}{2} \cos (4 \phi-2 \omega)+\frac{1}{2} \cos 2 \omega\right]
\end{aligned}
$$

In order that $i_{1}$ and $u_{1}$ be boundec ve must set

$$
\begin{aligned}
& \text { (3.13a) } \frac{d i_{D O}}{d \bar{c}}=0 \\
& \text { (3.13b) } \frac{d \varepsilon_{0}}{d \bar{d}}=0 \\
& \text { (3.13c) } \frac{d \omega_{0}}{d \bar{\phi}}=-\frac{\cos ^{4} i_{00}}{2 p^{4}}\left(1-5 \cos ^{2} i_{\infty}\right)=s_{0}
\end{aligned}
$$

Note the similarity of $(3.13 a)$ and $(3.13 c)$ to (2.22) and (2.23) establishing the analogy between $\alpha$ and $B$ of the modei equation with $i$ and w respectively for the main problem

Thus, the elements to first order become

$$
\text { (3.14) } e_{0}=\text { const. } \quad i_{\infty 0}=J_{0}=\text { const. } \quad \omega_{0}=s_{0}+\omega_{\infty}
$$

where $w_{\infty}$ is a constant depending upon the initial conditions.

Equations (3.12) can now be solved to gire

$$
\text { (3.15a) } \begin{aligned}
i_{1} & =\frac{1}{2 p^{4}} \cos ^{5} i_{00} \operatorname{sini} i_{\infty 0}\left[\cos 2 \phi+e_{0} \cos (\phi+w)+\frac{e_{0}}{3} \cos (30-w)\right] \\
(3.15 b) \quad u_{1} & =\frac{\cos ^{6} i_{00}}{2 p^{6}}\left[-1+3 \cos ^{2} i_{\infty}-\frac{e_{0}^{2}}{2}\left(1-5 \cos ^{2} i_{\infty 0}\right)\right.
\end{aligned}
$$




$$
\begin{aligned}
& +\frac{e_{0}^{2}}{4 \cdot}\left(1-3 \cos ^{2} i_{\infty}\right) \cos 2 w-\left(\frac{1}{3} \sin ^{2} i_{\infty}-\frac{e_{0}^{2}}{3}+\frac{5}{6} e_{0}^{2} \sin ^{2} i_{\infty}\right) \cos 2 \\
& +\frac{e_{0}^{2}}{6}\left(1-9 \cos ^{2} i_{\infty}\right) \cos 2(4-\omega)-\frac{e_{0}}{12}\left(5-11 \cos ^{2} i_{\infty}\right) \cos (34-\omega) \\
& \left.-\frac{e_{0}^{2}}{12}\left(1-3 \cos ^{2} 1\right) \cos (44-24)\right)
\end{aligned}
$$

To $O\left(\varepsilon^{3 / 2}\right)$ all the forcing terms on the right-hand sides of the eqantioms far $u_{3 / 2}$ must be removed for boundedness, biving

$$
\begin{aligned}
& \frac{d i_{01 / 2}}{d i}=0 \quad \frac{d e_{1 / 2}}{d \bar{l}}=0 \quad \frac{d w_{1 / 2}}{d i}=s_{1} i_{01 / 2} \\
& s_{n}=\frac{d^{n} s_{0}}{d i_{\infty}}, n=1,2, \ldots
\end{aligned}
$$

which implies that

$$
\text { (3.17) } i_{01 / 2}=j_{1 / 2}=\text { constant } \quad e_{1 / 2}=\text { constant } \quad \omega_{1 / 2}=s_{1} j_{1 / 2}+w_{1 / 20}
$$
were $v_{1 / 20}$ is a constant depending on the initiel condition.

The requirement that $i_{2}$ and $u_{2}$ be bounded provides the folloving equations ror 1,1 , and $e_{1}$ :

(3.18s) $\frac{d f_{01}}{d \bar{\varphi}}=c_{2} \sin 2 w$

$(3.18 b)$

$$
\frac{d w_{1}}{d i}=\frac{1}{2} s_{2}\left(i_{01 / 2}\right)^{2}+s_{1} 1_{01}+A_{0}+A_{2} \cos 20
$$

$(3.18 c) \quad \frac{d e_{1}}{d i}=B_{2} \sin 2 w$ 
The solutions of $(3.16)$ subject to the iutial conditions

$$
\text { (3.19) } \quad *=v \quad e_{n}=n_{n} \quad y_{n}
$$

et $t=$ are

(3.20a) $1_{01}=j_{1}+\frac{c_{2}}{2 s_{0}}(\cos 2 w-\cos 2 w)$

$(3.20 b) \quad e_{1}=1+\frac{B_{2}}{2 S_{0}}(\cos 2 v-\cos 2 w)$

(3.20c) $\frac{d w_{1}}{d j}=\frac{1}{2} s_{2} j_{i / 2}{ }^{2}+s_{1}\left[j_{1}+\frac{c_{2}}{2 s_{0}}(\cos 2 w-\cos 2 w)\right]+A_{0}+A_{2} \cos 2 w$

The non-uniformities of the outer solution near $s_{0}=0$ are exhibited above and are a consequence of the non-validity of the expansions assumed in (3.8) near the critical inclination.

\subsection{Inner expansion}

Ao show in Section 2, the expansion procedure for inclinations close to the critical value should be of the form

$$
\begin{aligned}
\text { (3.21a) } u(\phi ; \varepsilon) & =\sum_{n=0} u_{n / 2}(\phi, \bar{\phi} ; \varepsilon) \varepsilon^{n / 2} \\
(3.21 b) \quad 1(\phi ; \varepsilon) & =\sum_{n=0} i_{n / 2}(\phi, \bar{\phi} ; \varepsilon) \varepsilon^{n / 2}
\end{aligned}
$$

shere

$$
\text { (3.2Ic) } \quad \bar{\phi}=\varepsilon^{3 / 2}=\varepsilon^{1 / 2}
$$

- Henceforth ell constants not defined in the text will be found in the Appendix with no additional reference. 
and ve are interested in the case where

(3.21d) $\quad s_{0}=\varepsilon^{1 / 2} \bar{s}_{0}$, vith $\bar{s}_{0}=0(1)$

Upon substitution of $(3.21)$ into $(3.7)$ we obtain the following equations for the leading terms:

(3.22a) $\frac{\partial^{2} u_{0}}{\partial \phi^{2}}+u_{0}=\frac{\cos ^{2} i_{0}}{p^{2}}$

$(3.22 b) \quad \frac{\partial i_{0}}{3 b}=0$

whose general solution is of the form:

$(3.23 a)$

$$
i_{0}^{*}=i_{0}^{*}(\overline{0 ; E})
$$

$(3.23 b)$

$$
u_{0}=\frac{\cos ^{2} i_{0}^{*}}{p^{2}}\left(1+e^{*}(\bar{\phi}, \varepsilon) \cos \left[\phi-\omega^{*}(\bar{\phi} ; \varepsilon)\right]\right.
$$

We also expanci the elesents of the inner soiution in the form:

$(3.24 a) \quad i_{0}^{*}(\bar{\phi} ; \varepsilon)=\sum_{n=0} i_{o n / 2}(\bar{\phi}) e^{n / 2}$

(3.24b) e $e^{*}(\bar{\phi} ; \varepsilon)=\sum_{n=0}=e_{n / 2}(\bar{\phi}) \varepsilon^{n / 2}$

(3.24c) $\omega(\bar{\phi} ; \varepsilon)=\sum_{n=0}^{\omega} \omega_{n / 2}(\bar{\phi}) e^{n / 2}$

Since the homogeneous solution to $0\left(\varepsilon^{1 / 2}\right)$ is already accounted for by the expansion of the elements, we find $u_{1 / 2}{ }^{\bullet}=i_{1 / 2} \bullet=0$ and can jerive the following equations for the terms of $O(c)$. 
(3.25e) $\frac{\partial^{2} u_{1}}{2 \phi^{2}}+u_{1}=-\frac{1}{p^{6}} \cos ^{6} i_{\infty} \cdot \sin ^{2} i_{\infty}\left[\cos 2 \phi+\frac{e_{0}}{3} \cos \left(3 \phi-\omega^{*}\right)\right]$

$+\frac{\cos ^{6} i_{\infty}}{p^{6}}\left(-\frac{1}{2}+\frac{1}{2} \cos ^{2} i_{\infty} "\right)\left[1+\frac{e_{0}^{2}}{2}+\frac{e_{0}^{2}}{2} \cos 2(-\infty)\right]$

$+\frac{\cos ^{6} i_{00}}{p^{6}}\left(3-7 \cos ^{2} i_{\infty}\right)\left[\left(1+\frac{e_{0}^{* 2}}{2}\right) \cos 2 \phi+e_{0}^{*} \cos (3 \phi-\infty)\right.$

$\left.+\frac{e_{0}^{2}}{4} \cos \left(4 \phi-2 \omega^{*}\right)+\frac{e_{0}^{* 2}}{4} \cos 2 \omega\right]-\frac{2}{p^{6}} \cos ^{8} i_{\infty}^{*}[2-\cos 2 \phi$

$\left.-\frac{e_{0}}{2} \cos (3 t-\infty)\right]-\frac{e_{0} \cdot \cos ^{6} i_{\infty O}}{2 p^{6}}\left(1-3 \cos ^{2} i_{\infty}\right)(-\cos (34-\omega)$

$\left.-\frac{e_{0}}{2} \cos \left(4 \phi-2 \omega^{*}\right)+\frac{e_{0}}{2} \cos 2 \omega^{*}\right]-\frac{e_{0}^{* 2}}{2 p^{6}} \cos ^{8} i_{\infty}^{*}\left[1-\cos 2\left(\phi-\omega^{*}\right)\right.$

$\left.-\cos 2 \phi+\frac{1}{2} \cos \left(4 t-2 \omega^{\circ}\right)+\frac{1}{2} \cos 2 \omega^{\circ}\right)$

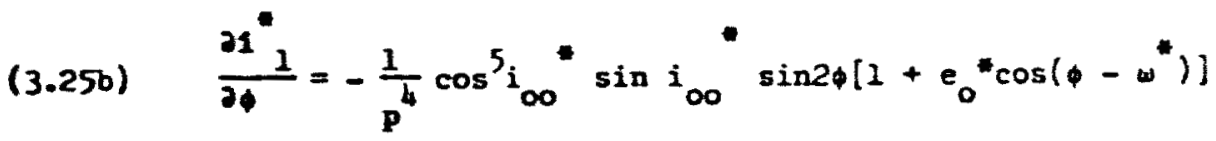

There are no terms proportional to $\sin$ or $\cos$ in (3.25a) and no terms wich depend on in $(3.25 b)$, so $(3.25)$ can be solved directly to yield

(3.26i) $\quad i_{1}=\frac{1}{2 p^{4}} \cos ^{5} i_{\infty} * \sin i_{\infty}^{*}\left[\cos 2 \phi+e_{0}^{*} \cos (\phi+\infty)+\frac{e_{0}^{*}}{3} \cos (3 \phi-\omega)\right]$

(3.26b) $\quad a_{1}=\frac{\cos ^{6} i_{\infty 0}}{2 p^{6}}\left[-1+3 \cos ^{2} i_{\infty} \cdot \frac{e_{0}^{2}}{2}\left(1-5 \cos ^{2} i_{\infty}\right)\right.$

$+\frac{e_{0}^{* 2}}{i}\left(1-3 \cos ^{2} i_{\infty}^{*}\right) \cos 2 \omega^{*}-\left(\frac{1}{3} \sin ^{2} i_{\infty}^{*}-\frac{e_{0}^{* 2}}{3}\right.$ 


$$
\begin{aligned}
& \left.+\frac{5}{6} e_{0}^{2} \sin ^{2} i_{\infty}\right) \cos 2\left(+\frac{e^{-2}}{6}\left(1-9 \cos ^{2} i_{\infty}\right) \cos 2(\phi-\infty)\right. \\
& \left.-\frac{e_{0}}{12}\left(5-11 \cos ^{2} i_{00}\right) \cos (34-v)-\frac{e_{0}^{-2}}{12}\left(1-3 \cos ^{2} i_{\infty}\right) \cos (44-24)\right)
\end{aligned}
$$

Since we are only interested in obtaining a solution correct to $O(c)$, we only give the boundedness conditions for the higher order terms.

Requiring ${ }^{*} 3 / 2$ and $i / 2$ to be bounded gires
$(3.27 a)$

$$
\frac{d i d}{d \bar{b}}=0 \quad(3.2 T b) \quad \frac{d e_{0}}{d \bar{t}}=0
$$$$
\frac{w_{0}}{d \bar{c}}=\bar{s}_{0}+s_{1} i_{01 / 2}
$$

and this inplies

$$
1_{\infty}=\text { contant }=1_{0}
$$$$
e_{0}^{-}=\text {constant }=n_{0}
$$

The boundedness of $u_{2}$ and $i_{2}$ tequires

(3.29a) $\frac{d i_{01 / 2}}{d \bar{t}}=c_{2} * \sin 2 w$

$(3.29 b)$

$$
\frac{d e_{1 / 2}}{d \bar{t}}=B_{2} \cdot \sin 2 \omega
$$

(3.29c) $\frac{d 1 / 2}{d \bar{c}}=\frac{1}{2} s_{2}\left(i_{01 / 2}\right)+s_{2} I_{01}+A_{0}+A_{2} \cos 2 \omega$

and finally in order to make $u_{5 / 2}$ and $i_{5 / 2}$ bounded ve wust set

$(3.30 a)$

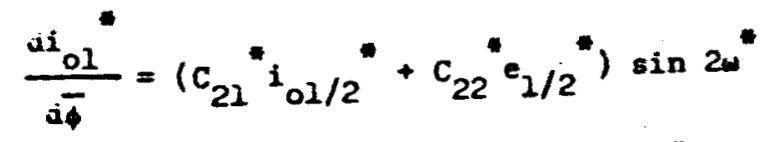

$(3.30 \mathrm{~b})$

$$
\frac{d e_{1}}{d \bar{d}}=\left[\bar{B}_{21} i_{01 / 2}+\bar{B}_{22} e_{1 / 2}-\frac{e_{0}}{4} \bar{s}_{0} \frac{\cos ^{4}{ }_{00}}{p^{4}}\left(1-3 \cos ^{2}{ }^{4}\right)\right] \sin 2 \omega^{*}
$$




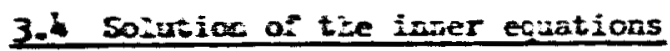

Pro equations (3.27c and $(3.29 a)$ re obtain

(3.31) $\frac{i_{01 / 2}}{\omega_{0}^{*}}=\frac{c_{2}^{*} \sin 2 *}{\bar{s}_{0}+s_{1} i_{01 / 2}}$

If the initial conditions are given as

(3.32)

$$
i_{\text {on }}^{*}=j_{n}^{*} \quad e_{n}^{*}=n_{n}^{*}
$$

at $t=1$, equation $(3.31)$ hes the solution

(3.33) $\quad i_{01 / 2} *=\frac{1}{s_{1}}\left[\left(\bar{x}_{0}-x_{1} \cos 2 \omega^{*}\right)^{1 / 2}-\bar{s}_{0}\right]$

mich upon substitution into (3.27c) gives

(3.34)

$$
\frac{a_{0}}{\sigma}=\left(\bar{x}_{0}-\alpha_{1} \cos 20^{*}\right)^{1 / 2}
$$

Dy we of equation (3.3h) and equation (3.29b) we now find

(3.35)

$$
c_{1 / 2}{ }^{\bullet}=\frac{B_{2}}{x_{1}}\left(\bar{x}_{0}-x_{1} \cos 2 \omega\right)^{\circ}+E_{1 / 2}
$$

Similarly, from equation ( $3.30 a)$ we calculate

$$
\begin{aligned}
& i_{01}^{*}=\frac{1}{x_{1}}\left[-c_{21} \cdot \frac{\bar{s}_{0}}{\bar{s}_{1}}+c_{22}{ }^{*} E_{1 / 2}\right]\left(\bar{\alpha}_{0}-\kappa_{1} \cos 2 \omega^{*}\right)^{1 / 2}-\frac{1}{2 s_{1}}\left[c_{21} *\right. \\
& +\frac{c_{22}}{c_{2}^{*} B_{2}^{*} ; \cos 2 \omega^{*}+I_{1}}
\end{aligned}
$$

Equation (3.30b) can next be integrated to

$$
\begin{aligned}
& \text { (3.37) } \quad e_{1}=\left(\bar{x}_{0}-x_{1} \cos 2 \omega\right)^{1 / 2}\left[-\frac{\bar{s}_{0}}{s_{1}} B_{21}^{*}+E_{1 / 2}{ }^{B}{ }^{*}-\frac{e_{0}}{4} \frac{\bar{s}_{0}}{p^{4}}(1\right. \\
& -3 \cos ^{2} 1_{\infty} i \cos ^{4}{ }^{\prime}=-\frac{1}{2}\left[\frac{B_{21}}{S_{1}}+\frac{B_{22}{ }^{*}{ }^{*}}{k_{1}}\right\} \cos 2 \omega+E_{1}
\end{aligned}
$$

34 
The solution of the apsialal motion will be considered in Section 3.6 .

\subsection{Matching and camposite expansions}

The problem of matching is essentialiy the same as the case discussed in Section 2 for the model equation. It must be remember that in the overlap domain, the initial conaitions are the same for both inner and outer expansions; thus

$$
i_{\infty}=i_{\infty}=j_{\infty}=j_{0}, \quad j_{n}^{*}=j_{n}, \quad e_{0}^{*}=e_{0}, \quad n_{n}^{*}=n_{n}, \quad w=w
$$

One can then calculate the following relations betreen the constants appearing in the inner and outer expansions:

$(3.39 a) \quad A_{0}=A_{0}-\frac{1}{2} s_{0}^{2}$

(3.39b) $\quad A_{2}^{*}=A_{2}-\frac{50}{4} \frac{\cos ^{4} i_{00}}{p^{4}}\left(1-3 \cos ^{2} i_{00}\right)$

(3.39c) $\quad B_{2}=B_{2}-\frac{S_{0}}{4} e_{0} \frac{\cos ^{4} i}{p^{4}}\left(2-3 \cos ^{2} i_{\infty}\right)$

(3.39d) $\quad c_{2}=c_{2}$

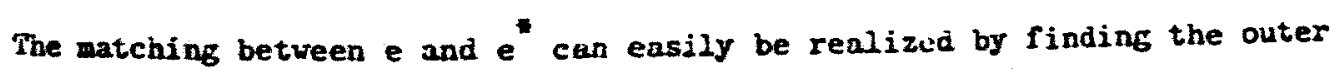
expansion of $e_{0}^{*}+\varepsilon^{1 / 2} e_{I / 2} *+\varepsilon e_{1}^{*}$. This is simply

$$
\begin{aligned}
& e_{0}^{*}+c^{1 / 2} e_{1 / 2}+c e_{i}^{*}=n_{0}^{*}+c^{1 / 2} n_{1 / 2}+\varepsilon n_{1}-\varepsilon \frac{B_{2}}{2 S_{0}}(\cos 2 \omega \\
& \left.-\cos 2 \psi^{*}\right)-\operatorname{cs}_{0} \frac{\cos ^{4} i}{4 p}\left(1-3 \cos ^{2} i_{00}\right)+0\left(\varepsilon^{3 / 2}\right)
\end{aligned}
$$


The last term of $O(E)$ in equation (3.40) arises from the outer expansion of $e_{1}$. Thus, $e_{0}^{*}+c^{1 / 2} e_{1 / 2}$ almost contain every term in tine outer expansion and the outer expansion of $e_{1}$ is mostly of higher order.

By comparing equation (3.40) vith the equation (3.20b), we note that in aodition to matching directly, the inner expansion contains the outer. It then folious that the composite expansion for e which is uniformly valid to ofel for all i 18

$$
e_{c}=e_{0}+\varepsilon^{1 / 2} e_{1 / 2}+c e_{2}
$$

The matching between $i$ and $i$ proceeds in a similar vay. The outer expansion of $t_{\infty}+e^{1 / 2} i_{01 / 2}$ is

$$
i_{00}+\varepsilon^{1 / 2} i_{01 / 2}=J_{0}+\varepsilon^{1 / 2} j_{1 / 2}+\varepsilon \frac{c_{2}}{2 S_{0}}(\cos 2 *-\cos 2 \omega)+0\left(\varepsilon^{3 / 2}\right)
$$

comparison of equation $(3.42)$ with equation $(3.20 \mathrm{a})$ shows that the inner expansion again contains the outer with the additional result that the outer expension of $i_{01}$ is $0\left(c^{3 / 2}\right)$. Thus, the composite expansion for $i_{0}$ is

$$
i_{o c}=i_{\infty}+e^{1 / 2} i_{01 / 2}+\varepsilon_{01}
$$

The above statements for and $i$ hold proviaed that w and $\omega$ are matched, this will be considered next.

From equations $(3.13 c),(3.16)$, and $(3.18 b)$, we have

$$
\frac{d \omega}{d i}=s_{0}+\varepsilon^{1 / 2} s_{1} J_{1 / 2}+\varepsilon\left[\frac{i}{2} s_{2} j_{1 / 2}{ }^{2}+s_{1} i_{01}+\Lambda_{0}+A_{2} \cos 2 \omega\right]+0\left(\varepsilon^{3 / 2}\right)
$$




$$
\begin{aligned}
\frac{d \omega}{d \phi} & =\bar{s}_{0}+s_{1}^{*} i_{01 / 2}=\varepsilon^{1 / 2}\left[\frac{z}{2} s_{2}\left(i_{01 / 2}\right)^{2}+s_{1} i_{01}+A_{0}\right. \\
& \left.+A_{2} \cdot \cos 2 \omega\right]+o(\varepsilon)
\end{aligned}
$$

According to equation (3.42) the outer expansion of $i_{01 / 2}$ 'contains $i_{01}$. Use of this result leads to the following outer expansion for $\frac{d \omega}{d \bar{\phi}}$ :

$$
\begin{aligned}
\frac{d \omega}{d \dot{b}} & =s_{0}+\varepsilon^{1 / 2} s_{1} J_{1 / 2} \cdot \varepsilon\left[\frac{1}{2} b_{2}\left(J_{1 / 2}\right)^{2}+s_{1} i_{01}+A_{0}\right. \\
& \left.+A_{2} \cdot \cos 2 \omega\right]+o\left(\varepsilon^{3 / 2}\right)
\end{aligned}
$$

Comparing equations (3.46) with $(3.44)$ we note that they are matched in any orerlap domain $s_{0}=O\left(\varepsilon^{\mu}\right)$ with $0<w<\frac{1}{2}$ because those terms not contained in the outer expansion of $d \omega / d \bar{w}$ have $s_{0}$ a factor (ef. Eq. 3.39) and are obviously small in the oreriap domain. The composite expansion for tine motion of the apse is therefore

$$
\text { (3.47) } \begin{aligned}
\frac{d \omega_{c}}{d \bar{c}} & =\bar{s}_{0}+s_{1} i_{01 / 2}+\varepsilon^{1 / 2}\left[\frac{1}{2} s_{2}\left(i_{01 / 2}\right)+s_{1} i_{01}+A_{0}\right. \\
& \left.+A_{2} \cos 2 \omega_{c}\right]+O(\varepsilon)
\end{aligned}
$$

uniformly to order $\varepsilon^{1 / 2}$ for all inclinations.

From the assuned foras for $u$ and $i$ it is easily seen that the uniforaiy valis expansions to $O(c)$ for all incalinations for these variables are

$$
u_{c}=\frac{\cos ^{2} i_{o c}}{p^{2}}\left[1+e_{c} \cos \left(--\omega_{c}\right)\right]+\varepsilon^{1 / 2} u_{1 / 2}+\varepsilon u_{1}
$$

$$
\text { (3.48b) } \quad i_{c}=i_{o c}+c^{1 / 2} i_{1 / 2}+c i_{1}
$$


wnere $i_{o c}, e_{c}$ and $\omega_{c}$ are used instead of $i_{0} e$ and $w$ in $u_{1 / 2}, u_{1}, i_{1 / 2}$ ano $i_{2}$ in equations $(3.48 a)$ and $(3.480)$ and $\omega_{c}$ can be obteined by integrating equatios $(3.47)$.

\subsection{Apsidal wotion}

The dominast behavior of the apsidal motion is described by the leading term. We have from $(3.34)$

$$
d \bar{\phi}=\left(\bar{x}_{0}-\alpha_{1}+2 \alpha_{1} \sin ^{2} \omega^{-1 / 2} d\right)^{*}+o\left(c^{1 / 2}\right)
$$

If we let

$$
\sin ^{2} \omega^{\circ}=v \quad 2 \sin \omega^{\circ} \cos \omega^{\circ}=\frac{d v}{d \omega}=2[v(1-v)]^{1 / 2}
$$

and consider only the leading term ve obtain

$$
\begin{aligned}
& \overline{-}-\bar{\phi}_{0}=\int_{0}^{v} \frac{\mathrm{d} \xi}{\left[\left(-8 \alpha_{1}\right)(\varepsilon-\lambda) \zeta(\xi-1)\right]^{\mathrm{i} / 2}} \\
& \lambda=\left(\kappa_{1}-\bar{\kappa}_{0}\right) / 2 \alpha_{1}
\end{aligned}
$$

For the eartb's potential the quantity $\kappa_{2}$ is positive near the criticai inclination. Thus the square root appearing in the above expression is reai only if

(3.52) $\quad v-\lambda>0, \quad \sin ^{2} \omega>\lambda$

- Because $c=4 / 7$ for the earth's potential. It is interesting to note that for Vinti's (i959) potentiki $c=5 / 18$ (cf. Shi (1963)) which impiing $k_{i}=0$ for the motion at the critical inclination. 
ifor ve have to aistinguish the following three cases:

\section{Case 1}

(3.53) $-x_{1}<\bar{x}_{0}<c_{1}$ or $0<\lambda<i$

In this case (3.50) becones an eliptic inteeral of the first kina.

$(3.54 a) \quad \overline{0}-\overline{0}_{0}=\left(2 x_{2}\right)^{-1 / 2} \vec{r}\left(x_{1}, x_{1}\right)$

where the amplitude $x_{1}$ is

(3.54b) $\quad x_{1}= \pm \tan ^{-1}\left[\frac{x_{1}+\bar{x}_{0}}{x_{1}-\bar{x}_{0}} \tan ^{2} \omega^{*}-1 j^{i / 2}\right.$

and the moduius is

$(3.54 c) k_{1}=\left[\left(\bar{x}_{0}+\alpha_{1}\right) / 2 x_{1}\right]^{1 / 2}$

Using elliptic functions we may express $\omega$ "explicitly as

(3.55)

$$
\omega^{*}= \pm \tan ^{-1}\left[\frac{\kappa_{1}-\bar{x}_{0}}{\kappa_{1}+\bar{x}_{0}}\left\{1+\operatorname{tn}^{2}\left[\left(2 \kappa_{1}\right)^{1 / 2}\left(\overline{1}-\bar{\phi}_{0}\right)\right] j\right]^{i / 2}\right.
$$

where the modulus of $t n$ is $k_{1}$.

The interpretation of this resuit is that the perigee performs a penduidis motion around $\pi / 2$ or $3 / 2$ with a maximum amplitude $\omega_{\max }^{*}= \pm \sin ^{-1} \lambda . \lambda$ aeperias on the initial conditions because after oubstituting the expression (i.2T) ior $\bar{x}_{0}$ ve obtain

(3.56) $\quad \lambda=\sin ^{2} w^{*}+\left(\bar{s}_{0}+s_{1} j_{1 / 2}\right)^{2} / 2 k_{1}$ 
Case 2

(3.57) $\bar{x}_{0}=x_{1}$ or $\lambda=0$

In this case we have

(3.58) $\quad-\bar{t}_{0}= \pm \int_{0}^{\omega} \frac{\mathrm{dE}}{\left(2 x_{1}\right)^{1 / 2} \cos E}=\frac{ \pm 1}{\left(8 x_{1}\right)^{1 / 2}} \log \frac{1+\sin \omega}{1-\operatorname{sin\omega }}$

or arter some manipulations

(3.59)

$$
\sin =-\frac{1-e^{ \pm\left(8 \alpha_{1}\right)^{1 / 2}\left(\bar{\phi}-\bar{\phi}_{0}\right)}}{1+e^{ \pm\left(8 x_{1}\right)^{1 / 2}\left(\bar{\phi}-\bar{\phi}_{0}\right)}}
$$

This means that $\omega$ approaches $O$ or asyoptotically as goes to infinity. This case represents the boundary between oscillatory and secular motion of the perigee. The boundary depends on the initial conditions. He have

$$
\lambda=\sin ^{2} w+\left(\bar{s}_{0}+s_{1} j_{1 / 2}\right) / 2 k_{1}=0
$$

which is possible only when the initial ralues

(3.61) $\mathbf{y}^{\bullet}=0$ or

and

(3.62) $\quad \bar{s}_{0}+s_{1} J_{1 / 2}{ }^{\bullet}=0$

are assumed. This means that initially the apse has to coincide vith the line or the nodes, and the inclination is exactly critical at least to the order kept in our calculations, because (3.62) is evidently the expansion of the Initial ralue of the small divisor. 


\section{Case 3}

(3.63) $\bar{x}_{0}>x_{1}$ or $\lambda<0$

In this case ve obtain

(3.64a) $\quad \bar{t}-\bar{t}_{0}=\left(\bar{x}_{0}+x_{1}\right)^{-1 / 2} r\left(x_{2}, x_{2}\right)$

vhere the modulus is

(3.64b) $\quad k_{2}=\left[x_{1} / x_{0}+x_{1}\right]^{1 / 2}$

and the anplitude is

(3.64c) $\quad x_{2}=\tan ^{-1}\left(\left[\left(\bar{x}_{0}+x_{1}\right) /\left(\bar{x}_{0}-x_{1}\right)\right]^{2 / 2} \tan 1\right)$

The nese of elliptic funetions gives

$$
\dot{*}=\tan ^{-1}\left[\left(\left(\bar{x}_{0}-x_{1}\right) /\left(\bar{x}_{0}+x_{1}\right)\right]^{1 / 2} \operatorname{tn}\left[\left(\bar{x}_{0}+x_{1}\right)^{1 / 2}\left(\bar{t}-\bar{t}_{0}\right)\right]\right\}
$$

where the modulus of to $k_{1} k_{2}$.

The apse angle eay assume any ralue in this case and the motion of the perigee is eccular. For large $\bar{k}_{0}, k_{2}^{2}$ beccues mall and ve was expand $F\left(x_{2}, k_{2}\right)$. This gives

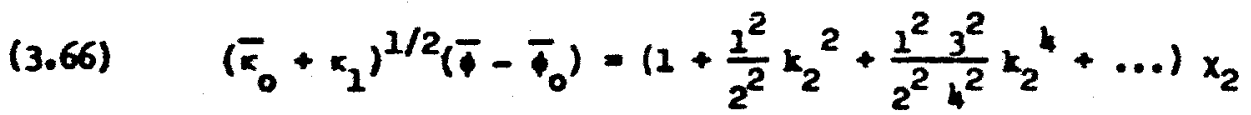

$$
-\frac{1}{8} k_{2}^{2} \cdot \sin 2 x_{2}-o\left(k_{2}^{k}\right)
$$

since $x_{2}+\oplus$ for large $\bar{x}_{0}$ (ef. (3.64c)) this shows that the notion of the perigee is secular vith soll edditional oscillations. In the previous discusaion of the beharior of the apsidal motion ve have considered only the 
solution of $\omega_{0}^{\bullet}$ A solution using all arailable information to $O\left(\varepsilon^{2}\right)$ must make use of the composite expansian as obtained in (3.47).

By substitution of $i_{0 i / 2}{ }^{\circ}$ and $i_{01}{ }^{\prime}$ in $(3.47)$, we obtain the folloving result which is uniforaly valid to $o\left(e^{1 / 2}\right)$ :

(3.67) $\frac{\mathrm{d}_{c}}{\overline{d \bar{c}}}=\left(1+c^{1 / 2} \bar{\sigma}_{1}\right)\left(\bar{x}_{0}-\alpha_{1} \cos 2 \omega_{c}\right)^{1 / 2}+\varepsilon^{1 / 2}\left[\delta_{0}+\delta_{2} \cos 2 \omega_{c}\right)$

Arter integration ve have

$$
\overline{0}-\bar{\phi}_{0}=\int_{0}^{\omega} c \frac{d \xi}{\left(1+\varepsilon^{1 / 2} \varepsilon_{1}\right)\left(\bar{x}_{0}-\kappa_{1} \cos 2 \xi\right)^{1 / 2}+\varepsilon^{2 / 2}\left[s_{0}+s_{2} \cos 2 \xi\right]}
$$

The evaluation of tinis integral leads to elliptic functions and a highly transcendental relation between $\omega_{c}$ and $\bar{\phi}$.

\subsection{Motion of the node}

Equation (3.6b) for the node can be brought to the rollowing form:

$$
\begin{aligned}
\frac{d \Omega}{d \phi} & =-\left[\frac{2}{p^{2}} u \cos ^{3} i \sin ^{2} \phi\right]-\varepsilon^{2}\left[\frac { 4 } { p ^ { 2 } } u ^ { 2 } \left(3 c u-7 c u \sin ^{2} i \sin ^{2} \phi\right.\right. \\
& \left.\left.-\frac{1}{p^{2}} \cos ^{4} i \sin ^{2} \phi\right)\right] \cos ^{3} i \sin ^{2} \phi+o\left(\varepsilon^{3}\right)
\end{aligned}
$$

Applying the composite expansions for and $i$ and substituting the know results ve obtain

- Note that $u_{1 / 2}$ and $i_{1 / 2}$ are zero. 


$$
\begin{aligned}
\frac{a}{\dot{\alpha} \phi} & =-\varepsilon\left[\frac{2}{p^{2}} u_{0} \cos ^{3} i_{o c} \sin ^{2} \phi\right]-\varepsilon^{2}\left\{\frac{2}{p^{2}} u_{i} \cos ^{3} i_{o c} \sin ^{2} \phi\right. \\
& -\frac{6}{p^{2}} u_{0} i_{1} \cos ^{2} i_{o c} \sin i_{o c} \sin ^{2} \phi+\frac{4}{p^{2}} u_{o}^{2}\left[3 c u_{0}-7 c u_{o} \sin ^{2} i_{o c} \sin ^{2} \phi\right. \\
& \left.\left.-\frac{i}{p^{2}} \cos ^{4} i_{o c} \sin ^{2} \phi\right] \cos ^{3} i_{o c} \sin ^{2} \phi\right\}+o\left(\varepsilon^{5 / 2}\right)
\end{aligned}
$$

Since ail quantities on the right-nand sicic of (3.70) are aiready known as functions of $\$$, the nocie coula be found by straightforward integration. liowever, for the sake of simplicity and a more systematic approach that avoids the shifting of orders of magnitude due to integration of long-period teras, ve vill also solve (3.70) by the two-variable expansion procedure.

We use the siou variable $\bar{\phi}=\varepsilon^{3 / 2}$ and assume the following expansion for the noce:

$$
\Omega=\frac{i}{\varepsilon} \sum_{n=0} \Omega_{n / 2}(0 . \bar{\varphi} ; \varepsilon) \varepsilon^{n / 2}
$$

ithe factor $\varepsilon^{-1 / 2}$ in front of the suration in $(3.7 i)$ is sugfested because the ieaaing term of the noaai velocity is of oracr $\varepsilon$ at all inclinations, which forces us to make the leading, term of the node itseif of order $\varepsilon^{-1 / 2}$ to insure that the derivative with respect to $\bar{\phi}$ be of order unity. Using the same procedure as for the other variables ve obtain the following equations:

$$
\text { (3.72a) } \quad \frac{\partial \Omega_{0}}{\partial \phi}=0
$$


unich implies tinat

(3.72b) $\quad \Omega_{0}=\Omega_{0}(\bar{b} ; \varepsilon)$.

Again, ve expand $\Omega_{0}$ in the form:

(3.73) $\quad \Omega_{0}(\bar{\phi} ; \varepsilon)=\sum_{n=0} \Omega_{\text {on } / 2}(\bar{\phi}) e^{n / 2}$

Since the right-hand side of equation (3.70) is $0\{\varepsilon\}$, we obtain

(3.74) $\quad \frac{\partial \Omega_{1 / 2}}{\partial \phi}=0 \quad$ and (3.75) $\quad \frac{\partial \Omega_{1}}{\partial \phi}=0$

implying that

(3.76) $\quad a_{1 / 2}=a_{1}=0$

because the integration constants are already included in the expansion (3.73).

Collecting the teras of order $\varepsilon$ we obtain

$$
\begin{aligned}
\frac{\partial \Omega_{3 / 2}}{\partial \phi} & =-\frac{\partial \Omega_{0}}{\partial \phi}-\frac{2}{p^{2}} u_{0} \cos ^{3} i_{00} \sin ^{2} \phi=-\frac{\partial \Omega_{0}}{\partial \phi}-\frac{\cos ^{5} i_{00}}{p^{4}}\left[1+e_{0} \cos \left(\phi-\omega_{c}\right)\right. \\
& \left.-\cos 2 \phi-\frac{e_{0}}{2} \cos \left(\phi-\omega_{c}\right)-\frac{e_{0}}{2} \cos \left(3 \phi-\omega_{c}\right)\right]
\end{aligned}
$$

The terms depending on $\bar{b}$ in (3.77) are $2 \Omega_{0} / \partial \bar{\phi}+\cos ^{5} i_{00} / p^{4}$. After substituting the expansion for $\Omega_{0}$ (the expansion for $i_{\text {oc }}$ has already been substituted in (3.77)), we require for poundedness

$$
\frac{\partial \Omega_{00}}{\partial \bar{C}}=-\frac{\cos ^{5} i_{00}}{p^{4}}
$$

The higher order terms in the expansion of $n_{0}$ and $i_{\text {oc }}$ are hence shifted to the next order. Integration of (3.78) gives 
(3.79) $\quad a_{\infty}=-\frac{\cos ^{5} i_{\infty 0}}{p^{4}}+i_{0}$

were $L_{0}$ is a constant depending on the initial conditions. The solution for $\Omega_{3 / 2}$ is

$(3.60)$

$$
\begin{aligned}
R_{3 / 2} & =-\frac{1}{p^{4}} \cos ^{5} i_{\infty}\left[-\frac{1}{2} \sin 2++e_{o} \sin \left(\phi-\omega_{c}\right)-\frac{e_{0}}{2} \sin \left(\phi+\omega_{c}\right)\right. \\
& \left.-\frac{e_{0}}{6} \sin \left(3 \phi-\omega_{c}\right)\right]
\end{aligned}
$$

in order to make $\Omega_{2}$ bounded, re must set

(3.81)

$$
\begin{aligned}
\frac{\partial \Omega_{01 / 2}}{\partial \bar{l}} & =5 \frac{i_{01 / 2}}{p^{4}} \cos ^{4} i_{\infty} \sin i_{\infty}=-\frac{5}{p^{4} s_{1}} \cos ^{4} i_{\infty} \sin i_{\infty 0}\left[\bar{s}_{0}\right. \\
& \left.-\left(\bar{x}_{0}-\alpha_{1} \cos \alpha_{c}\right)^{1 / 2}\right]
\end{aligned}
$$

or

(3.02)

$$
i_{0 i / 2}=-\frac{j}{p^{4} j} \cos ^{4} i_{\infty} \sin i_{\infty}[\bar{\phi}-\omega]+I_{1 / 2}
$$

vinere $L_{i / 2}$ is in integration constant and " is given by (3.55), (3.59) or (3.65) depending on the values of $\bar{\alpha}_{0}$ and $\alpha_{1}$.

The terms or $0\left(\varepsilon^{2}\right)$ depending on $\overline{0}$ anil are: $a n_{0 i} / 2 \bar{\phi}+\alpha_{0}+d_{2} \cos 2 \omega_{c}$

$+\left(5 / 2 p^{4}\right) \cos ^{\overline{3}} i_{\infty}\left(t-5 \cos ^{2} i_{\infty}\right)\left(i_{01 / 2}\right)^{2}-\left(5 / p^{4}\right) i_{01}{ }^{*} \cos ^{4} i_{\infty} \sin ^{i} i_{\infty}$

The boundedness requirement on $\Omega_{5 / 2}$ implies, after substituting for $i_{01 / 2}$ and $i_{01}$. that

(3.63) $\frac{\partial \Phi_{01}}{\partial \bar{\phi}}=D_{0}+D_{1}\left(\bar{\alpha}_{0}-\alpha_{1} \operatorname{sen} 2 \omega_{c}\right)^{1 / 2}+D_{2} \cos 2 \omega_{c}$

45 
The integration of (3.83) yields

(3.84) $\quad \Omega_{01}=D_{1} \overline{1}+\int_{0}^{\omega} c \frac{D_{0}+D_{2} \cos 2 \xi}{\left(\bar{x}_{0}-\alpha_{1} \cos 2 \xi\right)^{1 / 2}} d \xi+L_{2}$

were $I_{1}$ is an integration constant and the integral depends on the values of $\bar{x}_{0}$ and $\kappa_{1}$. The evaluation of this integral leads to eiliptic functions of the first and second kind and will not be exhibited here. 


\section{References}

Browier, D. 2959, Astron. J. 64, 9, 378 .

Cole, J. D., and Kevorkian, J. 1963, "Unifomly Val1d Asymptotic. Approcinations for Certain Non-inear Different 1al Equations," Proceedings a the Interationsl Symposiur on Mon-inear Mecianica and Eca-1snear Differential Equzions, Aug. 196́l, Ed. by J. P. IaSalle and S. Iefscheti, (Acaderaic Press, few York), p. 113.

Carfinkel, B. 1960, Astron. J. $65,10,624$.

Gröbner, W., and Hofrelter, i. 1949, "Integraltafel," V. 1 (SpringerVerlag, Viense and Innsoruck).

Ixsak, I. G. 1963, "On the Critical Irelpration in Satellite Tneory," Proceedings of the First Interitional Sysposium on the use of Artificial Sateliites For Geodesy, April 1962, Ed. by G. Vefs, (Horth-Holland Pub. Co., Amsteriam), p. 17.

Fiori, G. 1960, Astron. J. 65, 5, 291.

Jeffries, H. 1959, The Earth," 4th Ed. (Cambridge UnIr. Press, Camionide), 1959.

Kaplun, S., and Iagerstram, P. A. 1957, J. Math. Mech. 6, 5, 585.

Kevorixian, J. 196́2, "The Two-Variable Expansion Procedure for the Approximate Solution of Certain Hon-linear Differential Equations," Iecture jotes of the 1962 Summer Institute in Dymaical Astroncmy (Yale infrersity), $114 \mathrm{p}$.

Iagerstrcm, P. A., and Kevorikian, J. 1963, J. Hécanique 2, 2, 189.

Hersman, W. A. 196́2, "Ihe Crltical Inclination Problem in Satellite Orbit Theory," MisA rechntcal Rep. R-148, 29 p.

Struble, R. A. 1960, J. Math. Anal. Appl., $1,3 \& 4,300$. 196́1, Arch. Rational Yech. Anal., I, 2, 87. 1962, J. Kath. \& Mech., 10, 5, 691.

Sh1, I. Y. 2963, "The Earti Potential for Trajectory Calculations and Analytic Stuảes," Douflas Aircraft Co., Inc. Rep. Si-420́20, 19 p.

Vint1, J. 1959, J. Res. Nat. Bur. Standardo Sect. B, 63B, 2, 105. 


\section{Mrrendix}

(Derinition or Constants)

(A.1)

$$
\begin{aligned}
A_{0}= & +\frac{\cos ^{8} 1_{00}}{2 p^{8}}\left[-\frac{27}{24}+\frac{2}{2}=-\frac{25}{40} e_{0}^{2}+\frac{27}{8} e_{0}^{2} c\right. \\
& +\left(\frac{17}{4}-54 c+\frac{27}{0} e_{0}^{2}-\frac{259}{4} e_{0}^{2} c\right) \cos ^{2} i_{\infty} \\
& \left.+\left(-\frac{85}{24}+\frac{147}{2} c-\frac{25}{10} e_{0}^{2}+\frac{557}{3} e_{0}^{2} c\right) \cos ^{4} i_{\infty}\right]
\end{aligned}
$$

(A.2)

$$
\begin{aligned}
A_{2}= & +\frac{\cos ^{8} 1_{\infty}}{2 p^{8}} i-\frac{1}{6}-\frac{3}{2} c+\frac{5}{24} e_{0}^{2}-\frac{15}{4} e_{0}^{2} c \\
& +\left(-\frac{1}{3}+12 c-\frac{14}{3} e_{0}^{2}+42 e_{0}^{2} c\right) \cos ^{2} i_{\infty} \\
& \left.+\left(\frac{5}{2}-\frac{21}{2} c+\frac{45}{8} e_{0}^{2}-\frac{289}{4} e_{0}^{2} c\right) \cos ^{4} i_{\infty}\right) \\
B_{2}= & +\frac{\cos ^{8} i_{\infty}}{2 p^{8}} e_{0}\left[-\frac{1}{2}-\frac{3}{2} c-\frac{2}{12} e_{0}^{2}+\frac{3}{2} e_{0}^{2}\right. \\
& +\left(-\frac{1}{3}+12 c+\frac{4}{3} e_{0}^{2}-12 e_{0}^{2} c\right) \cos ^{2} 1_{\infty} \\
& \left.+\left(\frac{5}{2}-\frac{21}{2} c-\frac{5}{4} e_{0}^{2}+\frac{21}{2} e_{0}^{2}\right) \cos ^{4} i_{\infty}\right)
\end{aligned}
$$

(A.3)

(A.4)

$$
\begin{aligned}
\bar{i}_{21}= & -\frac{e_{0}}{2 p^{8}} \cos ^{7} i_{\infty 0} \text { ain } 1_{\infty}\left[-\frac{4}{3}-12 c-\frac{2}{3} e_{0}^{2}+12 e_{0}^{2} c\right. \\
& +\left(-\frac{10}{3}+120 c-\frac{40}{3} e_{0}^{2}-120 e_{0}^{2} c\right) \cos ^{2} i_{\infty 0} \\
& \left.+\left(30-126 c-15 e_{0}^{2}+126 e_{0}^{2} c\right) \cos ^{4} i_{\infty}\right]
\end{aligned}
$$


(A.5)

$$
\begin{aligned}
& B_{22}=\frac{\cos ^{8} 100}{2 p^{8}} l-\frac{1}{6}-\frac{3}{2} c-\frac{1}{4} e_{0}^{2}+\frac{9}{2} e_{0}^{2} c \\
& +\left(-\frac{1}{3}+22 c+4 e_{0}^{2}-36 e_{0}^{2} c\right) \cos ^{2} 1_{\infty} \\
& \left.+\left(\frac{5}{2}-\frac{21}{2} c-\frac{15}{4} e_{0}^{2}+\frac{63}{2} \operatorname{ce}_{0}^{2}\right) \cos ^{4} 1_{\infty}\right) \\
& \left.+(30-252 c) \cos ^{4} i_{\infty}\right]
\end{aligned}
$$

(A.Bj $\quad G_{22}=\frac{1}{2} \frac{e_{0}}{p^{8}} \cos ^{9} 1_{\infty}$ in $1_{\infty}\left[-\frac{1}{6}+3 c+\left(\frac{5}{2}-21 c\right) \cos ^{2} i_{\infty}\right]$

(A.9) $\quad A_{0}^{*}=+\frac{\cos ^{8} i_{\infty}^{*}}{2 p^{8}}\left[-\frac{23}{24}+\frac{2}{2} c-\frac{25}{48} e_{0}^{* 2}+\frac{27}{8} e_{0}^{*^{2}} c\right.$

$+\left(\frac{27}{4}-54 c+\frac{21}{8} e_{0}^{* 2}-\frac{189}{4} e_{0}^{* 2} c\right) \cos ^{2} i_{\infty}^{*}$

$\left.+\left(-\frac{235}{24}+\frac{147}{2} c-\frac{15}{16} e_{0}^{* 2}+\frac{567}{8} e_{0}^{* 2} c\right) \cos ^{4} 1_{\infty}^{*}\right]$

(1.20) $h_{2}^{*}=+\frac{\cos ^{8} 1 \omega_{0}^{*}}{2 p^{8}}\left[+\frac{1}{12}-\frac{3}{2} c+\frac{5}{24} e_{0}^{*}-\frac{15}{4} e_{0}^{*^{2}} c\right.$

$+\left(-\frac{7}{3}+12 c-\frac{14}{3} e_{0}^{*^{2}}+42 e_{0}^{*^{2}} c\right) \cos ^{2} 1_{\infty}$

$\left.+\left(\frac{25}{4}-\frac{21}{2} c+\frac{45}{8} e_{0}^{*}-\frac{189}{4} e_{0}^{* 2} c\right) \cos ^{4} 1_{\infty}^{*}\right]$

so 
(A.21)

$$
\begin{aligned}
B_{2}^{*}= & +\frac{\cos ^{\bar{\delta}} i_{\infty}{ }^{*}}{2 p^{8}} e_{0}^{*} 1+\frac{1}{12}-\frac{3}{2} c-\frac{1}{12} e_{0}^{*^{2}}+\frac{3}{2} e_{0}^{*^{2}} c \\
& +\left(-\frac{T}{3}+22 c+\frac{4}{3} e_{0}^{*^{2}}-12 e_{0}^{* 2} c\right) \cos ^{2} 1_{\infty 0}^{*} \\
& +\left(\frac{25}{4}-\frac{21}{2} c-\frac{5}{4} e_{0}^{* 2}+\frac{21}{2} e_{0}^{*} c\right) \cos ^{4}{ }_{\infty}^{*}
\end{aligned}
$$

(A.12)

$$
\begin{aligned}
& B_{21}^{*}=-\frac{e_{0}^{*}}{2 p^{8}} \cos ^{7} 1_{\infty}^{\prime \prime} \sin i_{\infty}^{*} i+\frac{2}{3}-12 c-\frac{2}{3} e_{0}^{* 2}+22 e_{0}^{* 2} c \\
& +\left(-\frac{\dot{7} 0}{3}+220 c+\frac{40}{3} e_{0}^{*^{2}}-200 e_{0}^{*^{2}} c\right) \cos ^{2} i_{\infty} * \\
& \left.+\left(75-120 c-15 e_{0}^{*^{2}}+120 e_{0}^{*^{2}} c\right) \cos ^{4} i_{\infty}{ }^{*}\right] \\
& \mathrm{B}_{22}^{*}=+\frac{\cos ^{8} 1_{\infty}^{*}}{2 p^{8}}\left[\frac{1}{12}-\frac{3}{2} c-\frac{1}{4} e_{0}^{*^{2}}+\frac{9}{2} e_{0}^{*^{2}} c\right. \\
& +\left(-\frac{7}{3}+22 e+4 e_{0}^{*^{2}}-36 e_{0}^{*^{2}} c\right) \cos ^{2}{ }_{10} \\
& \left.+\left(\frac{25}{4}-\frac{21}{2} c-\frac{15}{4} e_{0}^{*^{2}}+\frac{63}{2} e_{0}^{*^{2}} c\right) \cos ^{4} 1_{\infty}^{*}\right)
\end{aligned}
$$

(A.13)

(A.14)

$$
c_{2}^{*}=+\frac{1}{4} \frac{e_{0}^{* 2}}{p} \cos ^{2} 1_{\infty}{ }^{*} \sin 1_{\infty}^{*}\left[-\frac{1}{6}+3 c+\left(\frac{5}{2}-21 c\right) \cos ^{2} i_{\infty}^{*}\right]
$$

(A.25)

$$
\begin{aligned}
c_{21}= & +\frac{1}{4} \frac{e_{0}^{*}}{p^{8}} \cos ^{8} 1_{\infty 0}^{*}\left(\frac{3}{2}-27 c-\left(\frac{175}{6}-261 c\right) \cos ^{2} 1_{\infty}^{*}\right. \\
& \left.+(30-252 c) \cos ^{4} 1_{\infty}^{*}\right]
\end{aligned}
$$

(A.16)

$$
\begin{aligned}
& \text { (A.16) } c_{22}^{*}=+\frac{1}{2} \frac{e_{0}^{*}}{p^{8}} \cos ^{9} 1_{\infty}^{*} \sin ^{1}{ }^{*}\left(-\frac{1}{6}+3 c+\left(\frac{5}{2}-21 c\right) \cos ^{2} 1_{\infty}^{*}\right) \\
& \text { (i.17) } s_{0}=-\frac{\cos ^{4} 1_{\infty}}{2 p^{4}}\left(1-5 \cos ^{2} 1_{\infty}\right)
\end{aligned}
$$

51 
(i..3B) $\quad s_{1}=+\frac{\cos ^{3} i_{\infty}}{2 p^{4}} \sin 1_{\infty 0}\left(2-15 \cos ^{2} i_{\infty}\right)$

(A.19) $\quad s_{2}=-\frac{\cos ^{2} i \infty}{p^{4}}\left(6-83 \cos ^{2} 1_{\infty}+90 \cos ^{4} i_{\infty}\right)$

(A.20) $\quad \bar{s}_{0}=\frac{1}{\varepsilon^{2 / 2}}\left(-\frac{\cos ^{4}{ }_{i}{ }^{*}}{2 p^{4}}\left(1-5 \cos ^{2} 1_{\infty}^{*}\right)\right.$ !

(A.21) $\bar{x}_{0}=\left(\bar{s}_{0}+s_{1} J_{1 / 2}\right)^{2}+x_{1} \cos 2 *^{*}$

(A.22) ${ }^{\prime}{ }_{1}=s_{1} c_{2}^{*}=+\frac{1}{8} \frac{e_{0}^{*^{2}}}{p^{12}} \cos ^{12}{ }_{1}{ }^{*} \sin ^{2} i_{\infty}{ }^{*}\left(2-15 \cos ^{2} 1_{\infty}{ }^{*}\right)$

$$
x\left[-\frac{1}{6}+3 c+\left(\frac{5}{2}-21 c\right) \cos ^{2} 1 \infty\right]
$$

(A.23) $\quad E_{1 / 2}=\eta_{1 / 2}{ }^{*}-\frac{B_{2}^{*}}{k_{1}}\left(\bar{s}_{0}+S_{1} j_{1 / 2}{ }^{*}\right)$

(A.24) $\quad E_{1}=\eta_{1}^{*}-\frac{\bar{s}_{0}+J_{1 / 2}{ }^{*} s_{1}}{k_{1}} l-\frac{\bar{s}_{0}}{s_{1}} B_{21}^{*}+E_{1 / 2} B_{22}{ }^{*}$

$$
\begin{aligned}
& \left.-\frac{e_{0}^{*}}{4} \frac{\bar{s}_{0}}{p^{4}}\left(1-3 \cos ^{2} 1_{\infty}^{*}\right) \cos ^{4} 1_{\infty}^{*}\right] \\
& +\frac{1}{2}\left[\frac{B_{21}^{*}}{S_{1}}+\frac{B_{22}^{*}}{{ }^{*}} B_{2}^{*}\right] \cos 2 v^{*}
\end{aligned}
$$

(A.25)

$$
\begin{aligned}
& I_{1}=J_{1}^{*}-\frac{1}{x_{1}}\left[-c_{21} * \frac{\bar{s}_{0}}{s_{1}}+c_{22}{ }^{*} E_{1 / 2}\right]\left(\bar{s}_{0}+j_{1 / 2}{ }^{*} s_{1}\right) \\
& +\frac{1}{2 S_{2}}\left[\mathrm{C}_{21}^{*}+\frac{\mathrm{C}_{22}{ }^{*}}{\mathrm{C}_{2}^{*}} \mathrm{~B}_{2}^{*}\right] \cos 2 \mathrm{v}^{*} \\
& \text { (A.26) } \lambda=\sin ^{2} w^{*}+\frac{\left(\bar{s}_{0}+S_{1} j_{1 / 2}\right)^{2}}{2 x_{1}}
\end{aligned}
$$

52 
(i.27) $\quad B_{0}=\frac{s_{2}}{2 s_{1}^{2}}\left(\bar{x}_{0}+\bar{s}_{0}^{2}\right)+s_{1} \bar{L}_{1}+a_{0}$

(i.28) $\quad g_{1}=-\frac{s_{2}}{s_{1}^{2}} \bar{s}_{0}+\frac{1}{1}=-\Sigma_{21} \bar{s}_{0}+c_{22} s_{2} E_{1 / 2}$

(i.29) $\quad \bar{s}_{2}=A_{2}-\frac{i}{2} i \frac{s_{2}}{s_{1}^{2}} c_{1}+c_{21}+\frac{c_{22}}{c_{2}} \bar{s}_{2}$

(A.30) $\quad \dot{a}_{0}=\frac{\cos ^{9} s c o}{p^{0}} ; \frac{2}{3}-\frac{9}{2} c+\frac{3}{b} e_{0}^{2}-\frac{2 \pi}{4} e_{0}^{2} c$

$$
+\left(-\frac{5}{6}+\frac{21}{2} c-\frac{5}{24} e_{0}^{2}+\frac{5}{4} e_{0}^{2} c\right) \cos ^{2} i_{00}=
$$

(A.31) $\quad a_{2}=\frac{\cos ^{9} 1_{\infty}}{p^{6}} e_{0}^{2}:-\frac{2}{3}+6 c+\left(\frac{5}{4}-\frac{21}{2} c\right) \cos ^{2} i_{\infty 0}$

(A.32) $\quad D_{0}=-a_{0}-\frac{5}{2} \frac{\cos ^{3} i_{00}}{s_{1}^{2} p^{4}}\left(4-5 \cos ^{2} i_{\infty 0}\right)\left(\bar{x}_{0}+\bar{s}_{0}^{2}\right)$

$$
+5 \frac{I}{p^{4}} \cos ^{4} 1_{\infty} \sin 1_{\infty}
$$

(A.33) $\quad D_{1}=+5 \frac{\cos ^{3} 1_{00}}{p^{4} s_{1}}\left[\left(4-5 \cos ^{2} 1_{\infty}\right) \frac{\bar{s}_{0}}{s_{1}}+\frac{\cos 1_{00} \sin 1_{\infty O}}{c_{2}}\left(-c_{21} \frac{\bar{s}_{0}}{s_{1}}\right.\right.$

$\left.+c_{22} E_{2 / 2}\right) j$

(A.34) $\quad D_{2}=-d_{2}-\frac{5}{2} \frac{\cos ^{3} 1_{00}}{s_{1} p^{4}}\left[\cos 1_{\infty} \sin 1_{\infty 0}\left(c_{21}+\frac{c_{22}}{C_{2}} B_{2}{ }^{*}\right)\right.$

$\left.-\left(4-5 \cos ^{2} t_{\infty}\right) c_{2}\right]$

53 


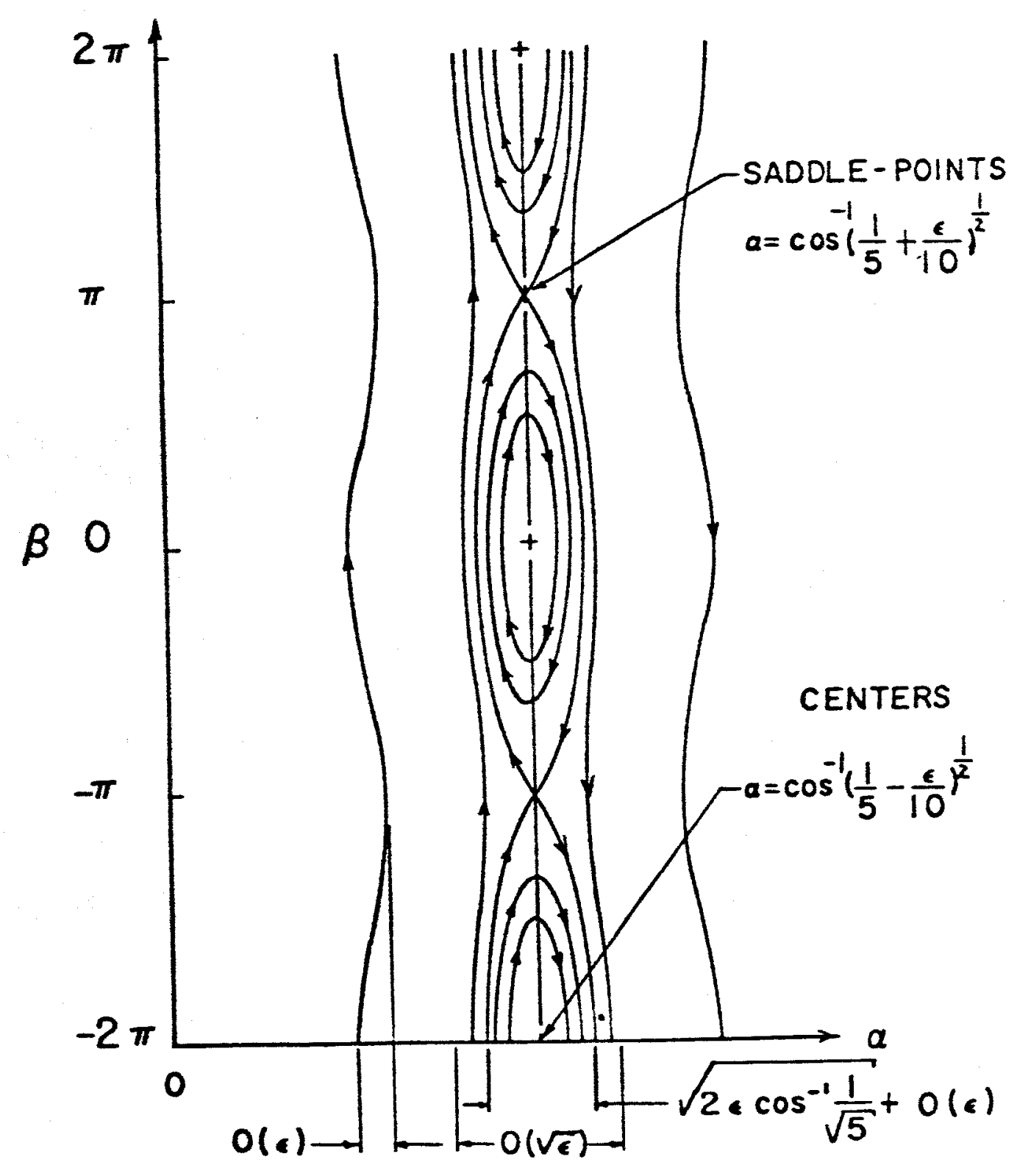

FIGURE 1 


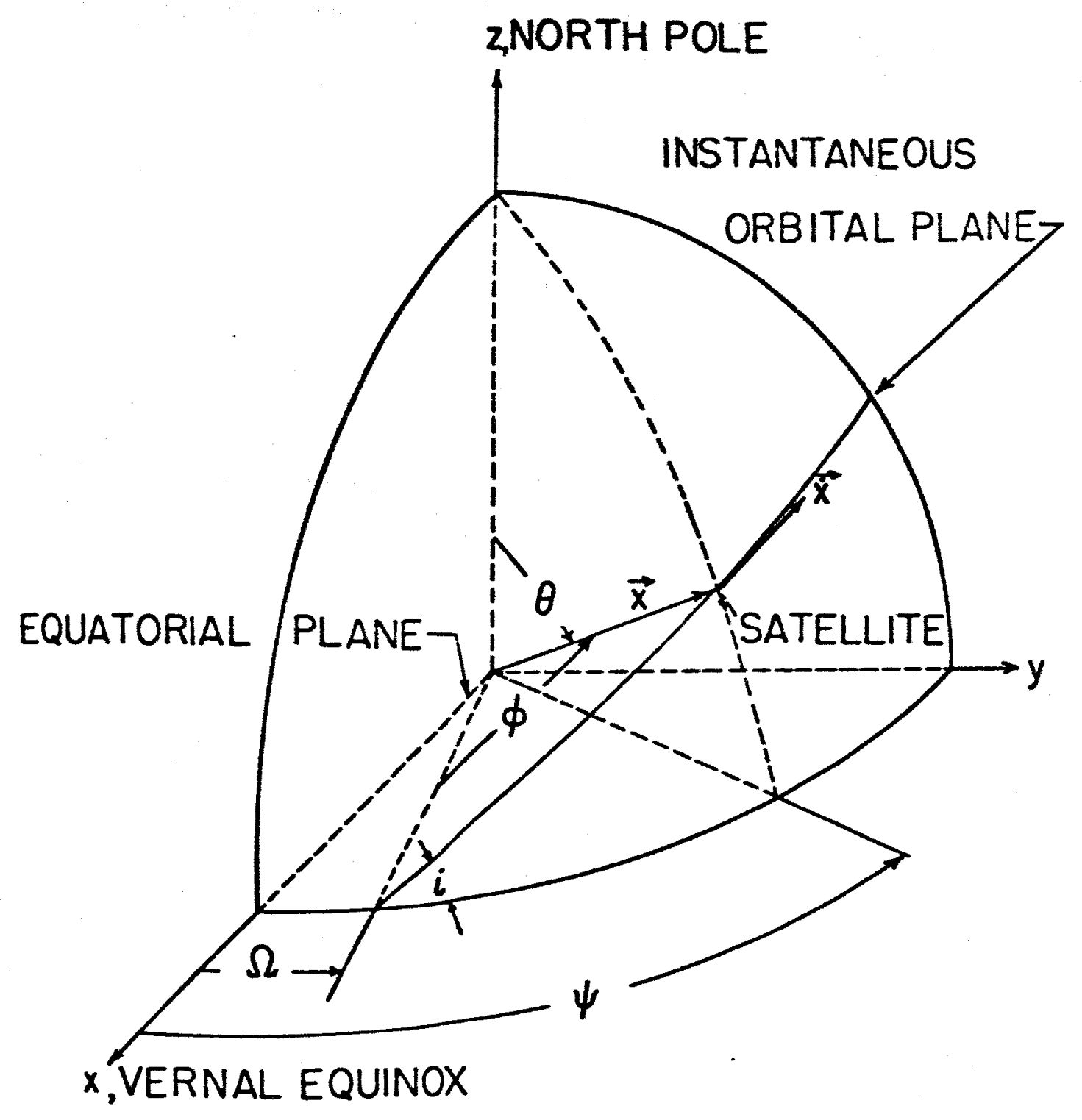

